Chapter 23

\title{
New Salivary Biomarkers of Human Exposure to Malaria Vector Bites
}

\author{
Papa M. Drame, Anne Poinsignon, Alexandra Marie, \\ Herbert Noukpo, Souleymane Doucoure, \\ Sylvie Cornelie and Franck Remoue
}

Additional information is available at the end of the chapter

http://dx.doi.org/10.5772/55613

\section{Introduction}

Mosquitoes are the most menacing worldwide arthropod disease vectors. They transmit a broad range of viral, protozoan and metazoan pathogens responsible of the most devastating human and animal diseases [1]. Among the main frequent mosquito-borne diseases, malaria represents the most widespread and serious infection in terms of heavy burden on health and economic development throughout the world. Despite substantial efforts and increasing international funding to eliminate it, malaria is still a major public health problem with nearly a million of deaths per year, especially in children younger than 5 years old (86\%) [2]. Approximately two thirds of the world's population live in areas at risk for malaria $[3,4]$. Understanding mechanisms that govern its transmission remains therefore a major scientific challenge, but also an essential step in the design and the evaluation of effective control programs $[5,6]$.

Entomological, parasitological and clinical assessments are routinely used to evaluate the exposure of human populations to Anopheles vector bites and the risk of malaria transmission. However, these methods are labor intensive and difficult to sustain on large scales, especially when transmission and exposure levels are low (dry season, high altitude, urban settings or after vector control) $[7,8]$. In particular, the entomological inoculation rate (EIR), the gold standard measure for mosquito-human transmission intensity of Plasmodium, is highly dependent on the density of human-biting Anopheles [9]. This latter is estimated by using trapping methods such as human-landing catches (HLC) of adult mosquitoes, the commonly used for sampling host-seeking mosquitoes and then for assessing the human exposure level. 
HLC may be limited because of ethical and logistical constraints to relevantly apply it to children [10]. Transmission estimates based on the prevalence or density of human infection are susceptible to micro-heterogeneity caused by climatic factors and the socioeconomic determinants of the host-seeking behavior [8]. Incidence of disease may be the closest logical correlate of the burden of disease on health systems. However, it can be subject to variability between sites and may not be appropriate for the evaluation of early phase studies of vector control or reliable for epidemic prediction [10]. More recently, serological correlates of transmission intensity have been described, yet they represent long-term rather than shortterm exposure data [8]. They are not then suitable in evaluating the short-term impact of vector control programs. Therefore, it is currently emphasized the need to develop new tools assessing reliably human malaria risk and control interventions, and monitoring changes over time at both population and individual levels $[5,6]$.

Malaria is a parasitic disease caused by protozoan agents of the genus Plasmodium (Aplicomplexa; Haemosporida). Five Plasmodium species are pathogen for humans: P. falciparum, P. vivax, P. ovale, P. malariae and P. knowlesi. During their complex life cycle in the female Anopheles mosquito (Insecta; Diptera), Plasmodium parasites go through several developmental transitions, traverse the midgut and reach the salivary gland (SG) epithelium. They acquire their maturity within SGs of the vector and can be then transmitted by the bite of the female mosquito. This latter needs, during the first days after emergence, to feed on sugar to meet the energy demands of basic metabolism and flight, but also to feed on vertebrate blood for its eggs' development and maturation [11], and therefore to keep perennial its life cycle and indirectly malaria transmission cycle.

Anopheles mouthparts comprise six pieces that form a long stylus allowing to perforate human tissues and to suck the internal liquid. However, it is clear that Anopheles mosquito acts not only as syringe injecting parasites during the bite. When taking a blood meal, it also injects into human skin avascular tissue [12] a cocktail of bioactive molecules including enzymes that are injected in human skin by saliva $[13,14]$. Some of these salivary compounds are essential to the Plasmodium life cycle [15]. They have substantial anti-hemostatic, anti-inflammatory, and immunomodulatory activities that assist the mosquito in the blood-feeding process by inhibiting several defense mechanisms of the human host [16]. Furthermore, many of them are immunogenic and elicit strong immune responses, evidenced by the swelling and itching that accompany a mosquito bite [17]. Specific acquired cellular [18, 19] or/and humoral responses are developed by human individuals when exposed to bites of Anopheles mosquitoes [20-23]. These immune responses may play several roles in the pathogen transmission ability and the disease outcomes [24]. In addition, recent studies have demonstrated that the intensity of the antibody response specific to salivary proteins could be a biomarker of the exposure level of human to Anopheles bites [22, 25]. Therefore, studying Anopheles-human immunological relationships can provide new promising tools for monitoring the real human-Anopheles contact and identifying individuals at risk of malaria transmission. It can also allow the development of novel methods for monitoring control and mosquito-release programmes' effectiveness. 
However, whole saliva could be inadequate as a biomarker tool, because it is a cocktail of various molecular components with different nature and biological functions. Some of these elements are ubiquitous and may potentially cause cross-reactivities with common salivary epitopes of other haematophagous arthropods [26]. In addition, a lack of reproducibility between collected whole Anopheles saliva batches has been observed and difficulties to obtain sufficient quantities needed for large-scale studies were highlighted [26]. Therefore, specific and antigenic proteins have been identified in the secretome of Anopheles mosquitoes and a specific biomarker of Anopheles bites was developed by coupling bioinformatic and immunoepidemiological approaches. This promising candidate, namely, the gSG6-P1 (An. gambiae Salivary Gland Protein-6 peptide 1), has been described to be highly antigenic [26]. It has been then validated as a pertinent biomarker assessing specifically and reliably the exposure level to Anopheles bites [27-29] and/or the effectiveness of malaria vector control [30] in all age-classes of human populations (newborns, infants, children and adults) from several malaria epidemiological settings (rural, semi-urban and urban areas...) throughout sub-Saharan Africa countries (Senegal, Angola and Benin).

The present chapter contributes therefore to a better understanding of the human-mosquito immunological relationship. It resumes most of the studies highlighting the roles of mosquito saliva on the human physiology and immunology, approaches, techniques, and methods used to develop and validate specific candidate-biomarkers of exposure to Anopheles bites and their applications on malaria control in several different epidemiological settings. Effects of various explanatory variables (age, sex, seasonality, differential use of vector control...) on human antibody responses to Anopheles salivary antigens are also discussed in the aim to optimize their use in epidemiological and vector-borne disease (VBD) control studies. Finally, different ways of application of such salivary biomarker of exposure of Anopheles vector bites in the field of operational research by National Malaria Control Programmes (NMCP) are highlighted.

\section{Human host-mosquito relationship: Roles of mosquito saliva}

Arthropods represent the vast majority of described metazoan life forms throughout the world, with species' richness estimated between 5 to 10 million [31]. The blood feeding habit has arisen and evolved independently in more than 14,000 species from 400 genera in the arthropod taxonomy [32]. In mosquitoes, only the adult female is hematophagous, whereas both male and female take sugar meals [33]. During the probing and the feeding stages, like all bloodsucking arthropods, female Anopheles must circumvent the highly sophisticated barriers represented by human defense systems (Fig. 1): haemostatic and inflammatory reactions, innate and adaptive immune system defenses. Therefore, they express in their saliva potent pharmacological and immunogenic components.

\subsection{Pharmacological properties of mosquito saliva}

The first-line of the human host non-specific defense to the insect bite is the haemostatic reaction. It provides an immediate response to the vascular injury caused by the intrusion of 


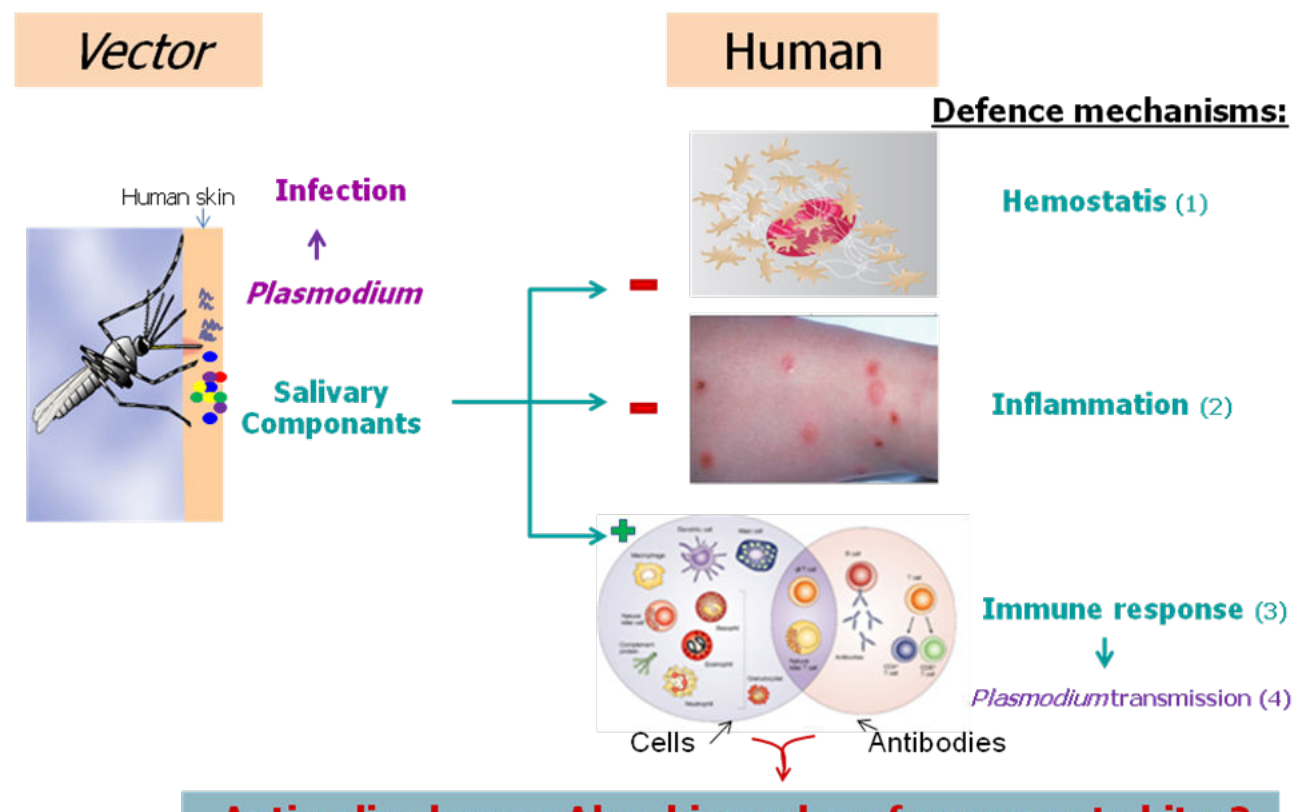

\section{Anti-saliva human $\mathbf{A b}=$ biomarker of exposure to bites?}

Figure 1. Effects of Anopheles saliva on hemostatic, inflammatory and immune reactions of the human to the vector bites.

the mosquito mouthparts in host vessels, thus preventing the extensive loss of host blood [32, 34]. The haemostatic reaction consists of three not physiologically distinct mechanisms: i) the blood coagulation that leads to the production of fibrin clots, ii) the thrombus formation and wound healing mediated by platelet aggregation, and iii) the vasoconstriction that leads to restricted influx of blood to the injured site. Each mechanism is activated by several pathways, in response to different exogenous and endogenous stimuli. Platelet aggregation is the first step in the haemostatic cascade and follows the interaction between blood platelets and the exposed extracellular matrix. This latter contains a large number of adhesive macromolecules such as collagen which is abundant underneath endothelial cells (not found in blood). This interaction results to the activation of platelets by mainly collagen and adenosine diphosphate (ADP, released by damaged cells and by activated platelets), the primary agonists of platelet aggregation. Platelets can be also activated by other agonists such as thrombin (produced by the coagulation cascade) and thromboxane $\mathrm{A} 2$ ( $\mathrm{TXA}_{2}$, produced by activated platelets) [35]. Activated platelets release endogeneous secretions such as serotonin and $\mathrm{TXA}_{2}$, two potent vasoconstrictors. In parallel, the blood coagulation mechanism is getting underway. The main task of the coagulation cascade is to produce fibrin that supports aggregated platelets in a thrombus formation. The coagulation process consists of an enzymatic cascade with two ways of activation, the exogenous and the endogenous, where several amplification points and regulatory mechanisms are known. 
However, mosquitoes can successfully engorge on their hosts within a half-minute because antihemostatic components of their saliva facilitate location of blood vessels and the blood sampling [36]. These salivary secretions, named sialogenins (from the Greek sialo, saliva; gen, origin, source; and ins for proteins), are mainly an array of potent anticoagulants, anti-platelets, vasodilators and anti-inflammatory substances [16, 32, 37, 38].

\subsubsection{Inhibition of platelet aggregation}

Compared to other blood-sucking arthropods like ticks and sand flies, only a limited number of Anopheles mosquito sialogenins involved in the inhibition of platelet aggregation have been characterized. Apyrase (Adenosine triphosphate (ATP)-diphosphohydrolase EC 3.6.1.5) is ubiquitous for hematophagous arthropods (mosquitoes, bugs, sand flies, fleas, triatomines, and ticks) and hydrolyses ATP and ADP into adenosine monophosphate (AMP) and inorganic phosphate $\left(P_{\mathrm{i}}\right)$, thus inhibiting platelet aggregation [16]. Three classes of apyrase have been characterized at the molecular level in different blood-sucking arthropods (reviewed by [39]). One named 5'-nucleotidase family is highly expressed in the salivary gland of Anopheles gambiae [40]. The D7 protein family is one of the most abundantly expressed sialogenins of mosquitoes. Two classes have been described in the saliva of mosquitoes: long (28-30 kDa) and short (15-20 kDa) forms [41-43]. The D7-related proteins may inhibit activation of host plasma. It has been described in Anopheles mosquitoes in a short form and may block the platelet activation by scavenging serotonin (agonist-positive feedback loop to increase platelet aggregation), while it principal function is reported to modulate tonus of vessels (vasoconstriction) [44]. Anophelin from An. stephensi saliva is a $30-\mathrm{kDa}$ protein that directly binds to immobilized collagen and specifically inhibits collagen-induced platelet aggregation and the intracellular $\mathrm{Ca}^{2+}$ increase [45]. It can also act by inhibiting the activity of thrombin which plays a role in concentration of platelet aggregation [46].

\subsubsection{Inhibition of blood coagulation cascade}

Arthropod anticoagulants mostly target factor X-active (fXa), which plays a central role at the nexus of the intrinsic and extrinsic pathways, as well as an ultimate role of thrombin in driving production of fibrin from fibrinogen. However, Anopheles mosquitoes produce an antithrombin [38]. In An. albimanus for example, Anophelin protein has been shown to be a potent anticoagulant that acts as a specific and tight-binding thrombin inhibitor [46], blocking or delaying then the clot formation process until blood meal completion [34]. In addition, a D7related protein of An. stephensi saliva has been characterized as an inhibitor of fXII [47].

\subsubsection{Vasodilator effect on host blood vessels}

In human, various types of endogenous vasoconstrictors (serotonin, $\mathrm{TXA}_{2}$, noradrenalin...) are released few seconds after tissue injury in order to stop the blood flow locally at the bite site. Diverse types of vasodilators have been characterized in the saliva of hematophagous arthropods. Aedes mosquitoes use sialokinins that mimic the endogenous tachykinin substance $\mathrm{P}$ which stimulate the production of nitric oxide (NO), a potent dilator of blood vessels [48, 49]. In contrast, the saliva of the adult female Anopheles mosquito has been shown to contain 
a myeloperoxidase with a vasodilator activity associated with a catechol oxidase/peroxidase activity [50]. This latter drives the $\mathrm{H}_{2} \mathrm{O}_{2}$-dependent destruction of noradrenalin and serotonin, two important endogenous vasoconstrictors [50]. In addition, some D7 proteins of Anopheles have been described to bind to biogenic amines such as serotonin, histamine, and norepinephrine [44]. These strategies remove the human host's ability to maintain vascular tone at the bite site, resulting to a weak but persistent local vasodilatation [14].

\subsection{Immunological effects of mosquito saliva}

The tissue injury causes an immediate onset of acute inflammation and innate immunity, which promote tissue repair, prevent colonization of the damaged tissues by opportunistic pathogens and initiates adaptive immunity, which is more specific [51]. These responses mobilize multiple elements such as phagocytes and antigen-presenting cells, cytokineproducing cells, T and B lymphocytes (TL and BL) and complement (classical and alternative pathways). It may result to the development of strong cell and humoral immune reactions, thereby altering physiologically the environment at the bite site and leading to the rejection of the blood-sucker [52]. The saliva of Anopheles mosquitoes (like blood-feeding arthropods in general) has selected, during evolution, compounds that can counter these host responses by modulating immune cells and cytokines' production [52,53]. This certainly allows mosquitoes to complete successfully a blood meal in only few seconds. Immunomodulatory effects of Anopheles mosquito saliva can therefore affect the transmission of pathogens and the development of associated pathologies [54]. Understanding the mechanisms which govern this immunomodulation could then allow the development of new prevention tools or strategies against malaria transmission [54-56].

\subsubsection{Inhibition of host inflammatory reaction}

The host inflammatory reaction following tissue injury consists of the triple response of Lewis: redness, heat and pain, triggering the awareness of the host to the blood sucker action [16]. If redness and heat are ones of the direct consequences of the dilatation of blood vessels, pain is induced by an increased vascular permeability under the effect of ADP, serotonin and histamine released by platelets and mast cells, following activation of the fXII by tissueexposed collagen [16]. The fXIIa converts prekallikrein to kallikrein, which hydrolyzes blood kininogen to produce the vasodilator peptide, bradykinin. This latter induces TNF- $\alpha$ (Tumor Necrosis Factor alpha) release by neutrophils [57], which in turn stimulates the release of IL (interleukin)-1 $\beta$ and IL-6 from various cell types. These cytokines contribute to the phenomenon of hyperalgesia (increased sensitivity to pain) that accompanies inflammation. Host inflammatory reaction to bites has been described as mast cells-dependent in individuals bitten by Anopheles mosquitoes [58]. In contrast to ticks which need to be attached to their host for several hours (tick Argasidx) or weeks (tick Ixodidx), mosquitoes take just few seconds for a successful blood meal. This certainly explains the poverty of anti-inflammatory components in their saliva in contrast to the ticks' one. Nevertheless, some salivary components of Anopheles mosquitoes can inhibit the human inflammatory reaction. In particular, a $16 \mathrm{kDa}$ D7 family proteins of An. stephensi (Hamadarin) inhibits the contact 
system by preventing the mutual activation between the fXIIa and the kallikrein in the presence of $\mathrm{Zn}^{2+}$ [47].

\subsubsection{Modulation of host immune response}

A role for arthropod saliva in modifying the outcome of transmission and infection is not a novel idea introduced in the context of mosquitoes and malaria parasites. The increased pathogen infectivity in association with ticks, sand flies, and mosquitoes saliva has been described previously [54]. If ticks that take a long time to engorge must additionally necessitate in their saliva anti-inflammatory and immunosuppressive factors, rapidly feeding dipterans, in particular mosquitoes and sand flies, clearly have evolved salivary factors that directly modulate host immune defenses [52]. One possible explanation is that these molecules have evolved because they have long-term beneficial effects for the populations rather than to the individual at the time of feeding [24]. Although the molecular mechanisms by which mosquito saliva induces alteration of the host immune response are unclear $[59,60]$, data evidently demonstrate that effects depend on the global regulation of the Th1/Th2 cytokines' balance, as it has been described in sand flies/Leishmania model, the most studied striking host-parasite vector system [61]. The Th1 response has been described to lead to a protective immunity and the resistance of the host to intracellular pathogens, while the Th2 response might favor the survivor of pathogens (parasites, virus...) and then the disease transmission and evolution [24]. For mosquitoes, studies have globally shown an enhancement of transmission and disease when pathogens are introduced in the presence of vector saliva. Mosquito saliva is commonly associated with a downregulation of the expression of Th1 and an upregulation of the Th2-type cytokines. In mouse models, mosquito saliva can potentiate the infection of arboviruses [24, 62, 63]. The co-inoculation of Sindbis virus with Aedes aegypti salivary gland extract resulted on a reduced interferon- gamma (IFN- $\gamma$ ) expression, when compared to injection of virus alone [64]. It has been also shown that $A e$. aegypti saliva contains multiple factors that can affect various components of the host immune response [65]. For example, factor Xa inhibitor may inhibit complement activation and leukocyte migration to the bite site [24] and other factors inhibit TNF- $\alpha$ release from activated mast cells [66]. Chickens subcutaneously infected with P. gallinaceum sporozoites in the presence of Aedes fluviatillis salivary gland homogenates showed a higher level of parasitaemia when compared to those that received only sporozoites [67]. For Anopheles, mice exposed to mosquito feeding in tandem with the inoculation of sporozoites had higher parasitemia and an elevated progression to cerebral malaria. This was associated with, in particular, elevated levels of IL-4 and IL-10, suppression of overall transcription in response to infection, and decreased mobility of dendritic cells and monocytes [19]. It was also described that Anopheles stephensi saliva downregulates specific antibody ( $\mathrm{Ab}$ ) immune responses by a mechanism that is mast cell and IL-10 -dependent [60]. IL-10, by inhibiting pro-inflammatory and Th1 cytokines, stimulates certain $\mathrm{T}$, mast and $\mathrm{B}$ cells and has pleiotropic effects in immunoregulation and inflammation, while IL-4 is the prototypical Th2 cytokine (it differentiates $\mathrm{CD} 4^{+} \mathrm{T}$-cells and up-regulates MHC class II production). The enhancement of IL-10 expression could account for reduction in secretion of other cytokines because it inhibits antigen presentation, IFN- $\gamma$ expression, and macrophage activation [68]. However, some 
data have suggested a paradoxical protective role of mosquito saliva against pathogen transmission and disease infection. Ae. aegypti saliva can inhibit infection of dendritic cells by dengue virus, and the pre-sensitization of dendritic cells with saliva prior to infection enhanced this inhibition. Moreover, the proportion of dead cells was also reduced in virusinfected dendritic cell cultures exposed to mosquito saliva, and an enhanced production of IL-12 and TNF- $\alpha$ was detected in these cultures [69]. In addition to these effects on cellular immunity, Anopheles saliva can also acts on humoral host immune response. Indeed, specific antibodies (immunoglobulins [Ig] G, M and E) to salivary antigens have been described in several studies $[20,22,23,25,56,70]$. However, the implication of these Ab responses in disease pathogenesis or protection is not yet elucidated.

Therefore, future studies are needed for an overall understanding of mosquito saliva effect, especially Anopheles mosquito saliva, in pathogen transmission, disease development and pathogenesis.

\subsubsection{Human host-Anopheles vector immune relationship and applications}

The study of immunological properties of salivary proteins of Anopheles mosquitoes represents a new research thematic which can significantly improve the understanding of Plasmodium transmission mechanisms and therefore help for the effective prevention and control of malaria. It can notably lead to major applications in three areas: i) development of vaccines, diagnosis, treatment, ii) prevention of allergies, and iii) development of biomarkers of exposure to bites and malaria disease risk.

The development of parasite transmission-blocking vaccines, by stimulating the immune response against the vector is an attractive alternative way for malaria control. Several studies targeted the effect of Abs specific to the mosquito midgut antigens have shown promising results [71-73]. The study of the immune response induced by vector saliva at the biting site and its potential effect on the transmission and the development of pathogens suggests the possibility to control parasite transmission by vaccinating the host with immunogenic salivary compounds [54, 74]. In a mouse model, it has been shown that two salivary proteins (29 and $100 \mathrm{kDa}$ ) of the female An. gambiae can induce production of Ab which can block about $75 \%$ of the invasion of An. stephensi salivary glands by P. yoelii sporozoites [75]. In addition, the prior exposition to non infective An. stephensi bites induces a Th1 immune response with increased production of IL-12 and IFN- $\gamma$. Its effect can subsequently limit future P. yoelii infection (reduced rate of liver and blood parasites) and the development of cerebral malaria in mouse [18]. In this context, saliva can be thought as a non-specific "adjuvant" which could be effective at inducing a Th1-biased environment that is known to be protective against malaria infection. However, the development of such vaccines is complex. For example, Ab produced by immunization (with salivary proteins) must be ingested by the mosquito during a bite, cross it midgut and digestive enzymes, migrate to the salivary glands, before they can block the invasion by sporozoites. Nevertheless, the possibility to develop a pan-arthropod vaccine has been recently demonstrated by another mechanism. Indeed, an immune response directed to salivary proteins that adsorb to pathogens can turn the microorganism into an innocent bystander of anti-salivary immunity as it has been recently reported in a salivary 
protein (Salp15) from the hard tick Ixodes scapularis [76] and vaccine candidate for the control of Lyme disease [77]. Unfortunately, any hematophagous arthropod saliva-based vaccine has not yet been tested on humans.

In the field of allergic reactions to salivary proteins of mosquitoes, the first studies were mainly conducted in Canada and Finland. They concerned Aedes and Culex mosquitoes which express a panel of allergens in their saliva during the blood feeding time [17, 56, 78]. These proteins can thus be used in recombinant form, as diagnostic tool of the level of human exposure to allergens or in immunotherapy injections for desensitization of human [56, 70, 79]. It exists yet no study highlighting the presence and effect of allergens in the Anopheles mosquitoes' saliva.

The study of immunological relationship between human-vector by quantifying specific $\mathrm{Ab}$ responses to salivary proteins may also allow the identification and characterization of biological markers for epidemiological assessment of the exposure of individuals and populations to the Anopheles bites and thus to the risk of malaria transmission [22]. The development of such biomarkers or indicators (see next chapter) can be a complementary alternative to current referent entomological and parasitological methods which present several limitations especially in low exposure/transmission contexts.

\section{Development of biomarkers of human exposure to Anopheles bites and indicators of malaria vector control effectiveness}

\subsection{Validation of concept with whole Anopheles saliva}

To improve the fight against malaria and regarding numerous limitations described with current entomological and parasitological tools, the World Health Organization (WHO) has emphasized the need of new indicators and methods to evaluate, at individual and population levels, the exposure level to Anopheles vectors and the effectiveness of vector control strategies. One promising concept is based on the fact that mosquito saliva injected to the human host during the vector bite is antigenic and can induce an adaptive humoral host response (see Figure 1). Therefore, a logical positive correlation between the human exposure level to Anopheles bites and human anti-mosquito saliva Ab level can be expected. In this way, antimosquito saliva $\mathrm{Ab}$ response can be a pertinent epidemiological biomarker of human exposure to vector bites.

The epidemiological importance of human exposure to the saliva of vectors has been firstly described in Lyme disease [80, 81], leishmaniasis [82] and Chagas disease [83]. During the last decade, studies have provided data on human exposure to anopheline saliva and its interaction with malaria transmission. In particular, Remoue et al. [22] have shown that children living in a seasonal malaria transmission region of Senegal developed IgG responses to An. gambiae whole saliva (WS). Interestingly, these specific IgG levels were positively associated with an increased rainfall and the Anopheles mosquito density, measured by referent entomological methods. Indeed, an increase in the level of IgG was observed according to the Anopheles aggressiveness and density in September (Figure 2), the peak of malaria transmission. 


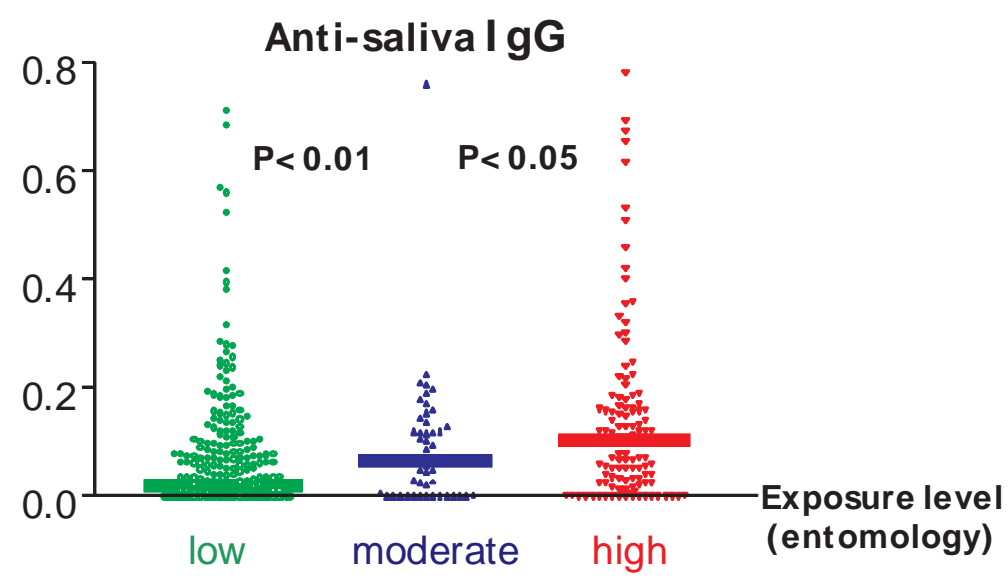

Figure 2. Anti-saliva IgG according to the intensity of exposure [22]. Individual absorbance (OD) values in September are shown for the three groups with different levels of exposure. Bars indicate the median value for each group. Statistical significances between each group by non-parametric Mann-Whitney U-test are indicated.

Importantly, IgG response to An. gambiae WS can predict clinical malaria cases. Indeed, children who developed a malaria attack in December had higher levels of anti-WS IgG in September of the same year, i.e. three months before they develop the disease (Figure 3) [22].

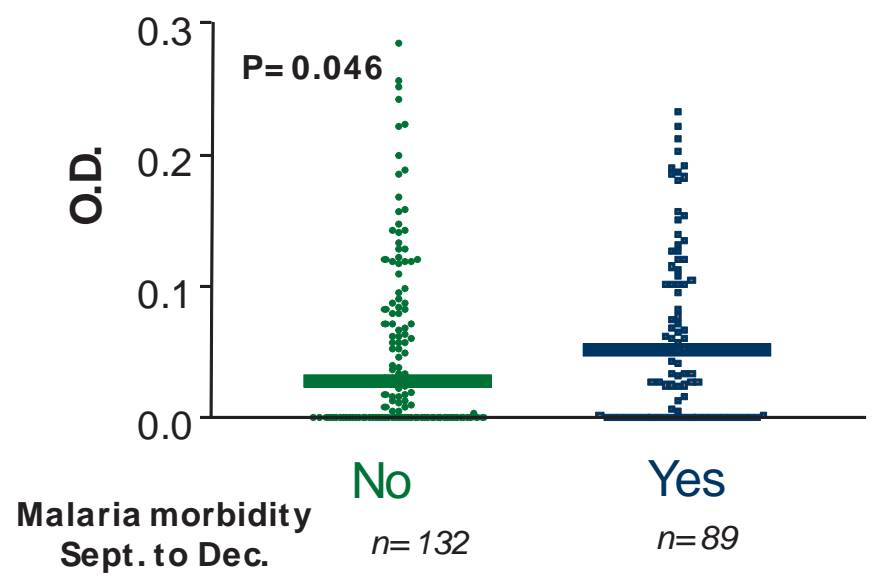

Figure 3. Anti-salivary lgG according to malaria morbidity. The results of individual absorbance (OD) values in September are shown according to subsequent detection of clinical malaria for the age $\geq 1$ year. Bars indicate the median value for each group. Statistical significance between groups is indicated by a non-parametric Mann-Whitney U-test).

Anti-mosquito saliva $\mathrm{Ab}$ appeared transitional. Soldier travelers transiently exposed to An. gambiae bites in endemic areas of Africa (especially Ivory Coast and Gabon) developed specific 
IgG responses to anti-An. gambiae WS which strongly decreased several weeks after the end of their trip [21]. In addition, anti-An. gambiae saliva IgG levels waned rapidly after 6 weeks of Insecticide-Treated Nets (ITNs) well-use in a semi-urban population in Angola, before a new significant increase two months later following the stop of ITN use [84]. Data on human exposure to anopheline saliva and its interaction with malaria were also provided by studies from other none African areas. In South-eastern Asia, it has been described that anti-An. dirus salivary protein $\mathrm{Ab}$ occur predominantly in patients with acute $P$. falciparum or $P$. vivax malaria; people from non-endemic areas do not carry such Abs [23]. In the Americas, the presence of anti-Anopheles saliva $\mathrm{Ab}$ has been also described. In adult volunteers from Brazil, anti-An. darlingi WS Ab levels increased with $P$. vivax infections [20]. The presence of anti-An. albimanus WS Ab with exposure to mosquito bite has been recently described in Haiti [25]. Specific IgG response to An. gambiae WS has also been described as an immunological indicator evaluating the efficacy of malaria vector control strategies. Indeed, Drame et al. have recently shown in a semi-urban area (Lobito, Provence Benguela) in Angola that specific IgG levels drastically decreased after the introduction of ITNs and this was associated with a drop in parasite load (Figure 4) [84].

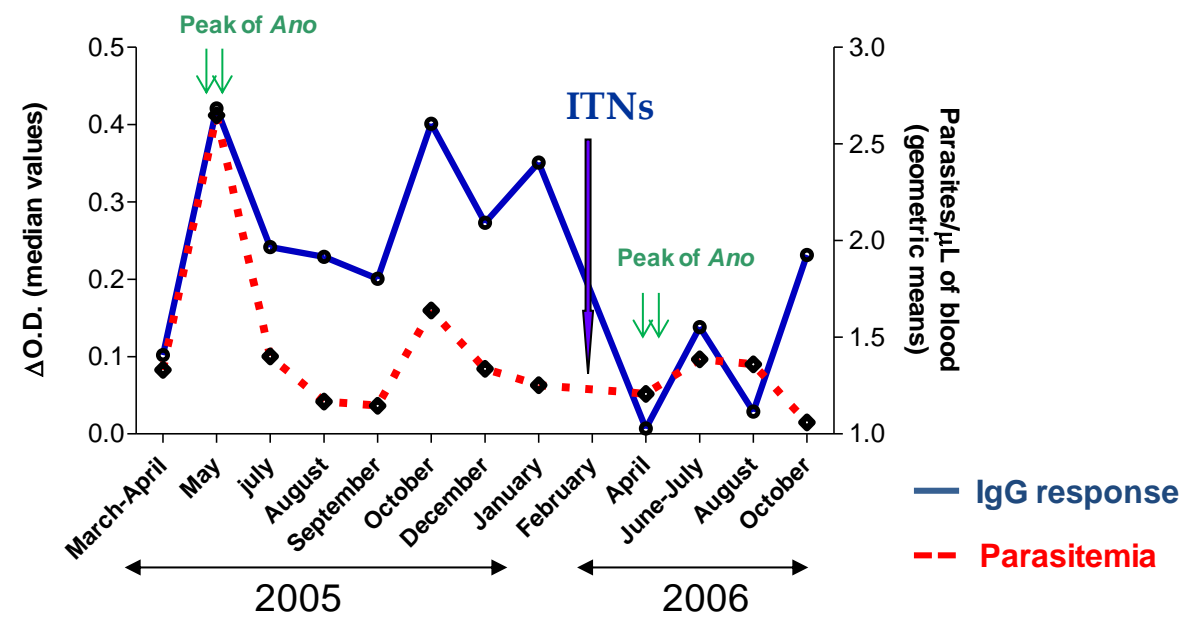

Figure 4. Evolution of anti-Anopheles gambiae saliva IgG and Plasmodium falciparum infections before and after ITN implementation, (Ano=Anopheles).

Anti-Anopheles saliva IgG response has also been recently used to evaluate and compare the effectiveness of three malaria vector control strategies in another area (Balombo) of Angola [85]. Indeed, Brosseau et al. [85] have investigated over a period of two years (2008-2009) Ab response to An. gambiae WS in children between 2 to 9 years old, before and after the introduction of three different malaria vector control methods: deltamethrin treated long lasting impregnated nets (LLIN) and insecticide treated plastic sheeting (ITPS) - Zero Fly®) (ITPS-ZF), deltamethrin impregnatedDurable(Wall)Lining(ITPS-DL-Zerovector $\left.{ }^{\circledR}\right)$ alone, and indoor residualspraying 
(IRS) with lambdacyhalothrin alone. They observed considerable decreases in entomological $(82.4 \%)$, parasitological $(54.8 \%)$ and immunological criteria analyzed. In particular, the immunological data based on the level of anti-saliva IgG Ab in children of all villages significantly dropped from 2008 to 2009, especially with LLIN+ZF and with IRS (Figure 5).

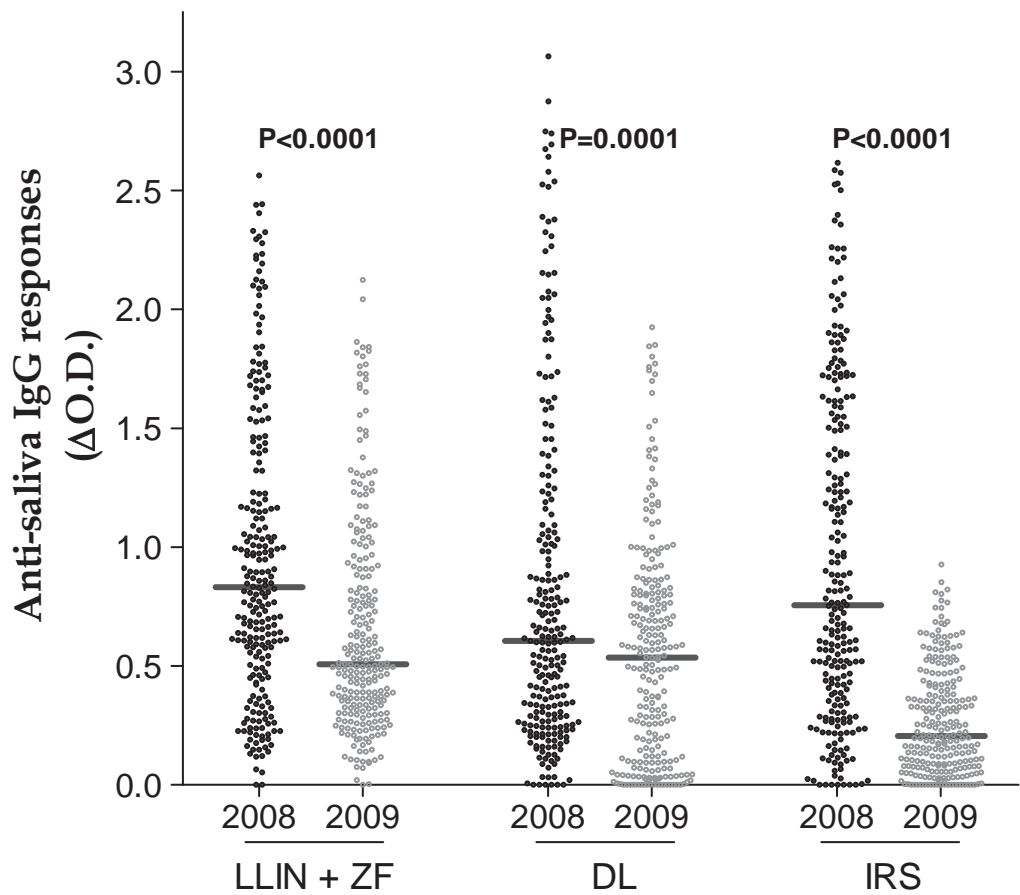

Figure 5. Comparison of median values of the $\lg G$ antibody response to Anopheles saliva obtained before and after implementation of each vector control method [85].

Taken together, these studies indicated that the estimation of human IgG Ab responses specific to Anopheles WS could provide a reliable biomarker for evaluating the Anopheles exposure level, the risk of malaria transmission, the disease outcomes and the effectiveness of vector control strategies. However, the pertinence and the practical large-scale application of serological tests for epidemiological purposes have been hampered by several limitations. First, WS is a cocktail of various molecular components with different nature and biological functions. Some components are Anopheles-specific and other widely distributed within genus, families, orders or classes of bloodsucking Diptera or Arthropods [16]. Therefore, the evaluation of Anopheles exposure or vector control effectiveness based on the immunogenicity of WS could be skewed and over or underestimated by possible cross-reactivities between common epitopes between mosquito species or other organisms [26]. Second, the collection of saliva or salivary gland extracts is tedious and time-consuming; therefore it will be difficult or impossible to have an adequate production of mosquito saliva needed for large-scale epidemiological studies [26]. 
Third, saliva composition can be affected by several ecological parameters such as age, feeding status or infectivity of Anopheles [86], which in turn may influence the anti-saliva immune response measured and may cause a lack of reproducibility between saliva batches. An alternative for optimizing the specificity of this immunological test would thus be to identify Anopheles genus-specific proteins [87].

\subsection{Methods for the identification of specific Anopheles salivary proteins}

The isolation of salivary components has been a challenge for many years. Many functional active salivary proteins have been isolated following classical biochemical and molecular biology approaches [88]. Protocols mainly consisted of the isolation of salivary components from hundreds of salivary gland pairs, obtaining amino-terminal or internal peptide sequence of the purified component, screening of a salivary gland library with the information obtained, and isolation of the cDNA or gene of interest (Fig. 6).

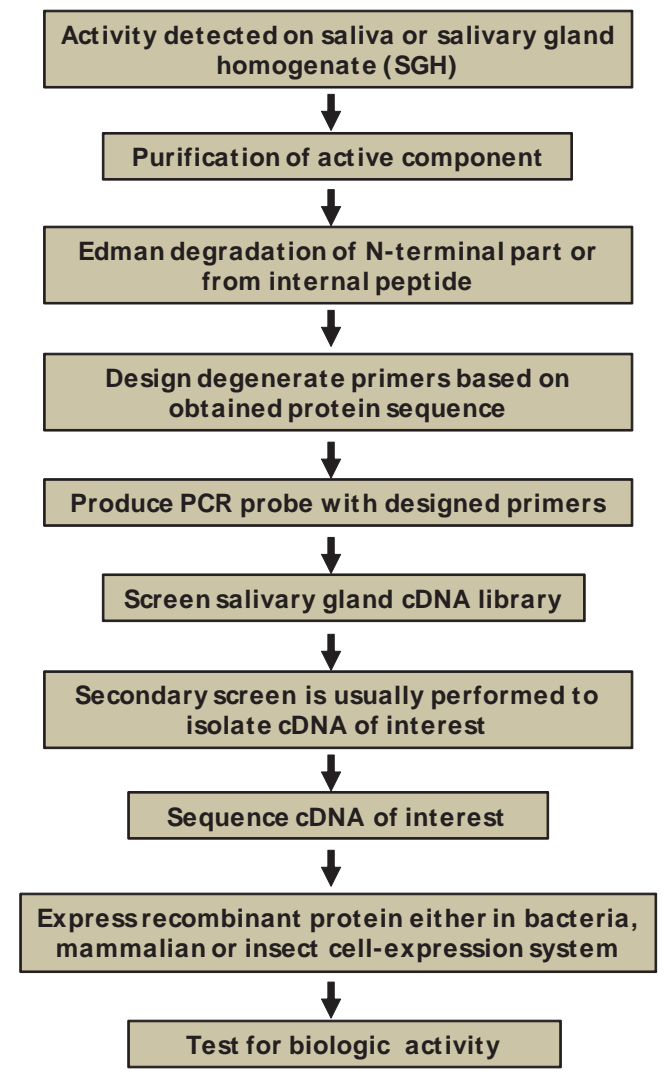

Figure 6. Classical biochemical and molecular biology protocol used for isolation and characterisation of salivary proteins and cDNA from vectors of disease [90]. 
During the last decade, technical advances in molecular biology have allowed the sequencing of the genome, including transcripts of salivary glands [89], of most disease vectors, comprising Anopheles mosquitoes [90]. However, protocols do not allow to obtain entire sequences [89]. Nowadays, researchers have switched from testing one salivary molecule at a time to studying the whole complex of genes and secreted proteins in blood-feeding arthropods using transcriptomic and/or proteomic approaches. The transcriptomic is the complete set of transcripts in an organism for a specific developmental stage or physiological condition. Transcriptomic techniques help to interpret the functional elements of the genome, and to understand the transmission and development of diseases [91]. They aim to catalogue transcript of major Anopheles species, including mRNAs, non-coding RNAs and small RNAs; to determine the transcriptional structure of genes and to quantify the changing expression levels of each transcript during development and under different conditions [91]. Proteomic is a large-scale study of the gene expression at the protein level, which ultimately provides direct measurement of protein expression levels [92]. The proteomic revolution is hitting the vector biology field as well as many other fields. The isolation and sequencing of all the proteins from SGs of disease vectors and, more specifically, secreted salivary proteins, is clarifying the complexity of proteins present in the saliva of various blood-feeding arthropods [93]. During the last years, a comprehensive high-throughput approach has been developed (Figure 7) [88]. It combines massive sequencing protocol of high quality full-length salivary gland cDNA libraries, a proteomic approach to isolate a large set of salivary proteins, and high-throughput computational biology and functional assays to analyze and test the biologic activities of these novel molecules. It is a powerful tool which can help easily and rapidly to identify and characterize genes or transcripts encoding for various proteins of SGs (the sialome) of blood-sucking arthropods. This high-throughput approach has then allowed an unprecedented insight into the complexity of salivary gland compounds of mosquito vectors of disease agents, indicating that the diversity of their targets is still larger than previously thought [16].

\subsection{Salivary proteins (sialome) of Anopheles mosquitoes}

The increasing power of large-scale genomic, transcriptomic and proteomic analyses allowed the accumulation of a considerable amount of information on the salivary secretions of bloodsucking arthropods [86]. As far as mosquitoes are concerned, the analysis of salivary transcriptomes of a number of Anopheles have allowed the discovery of a variety of genes that matched the sequence of various protein families, providing some clues on the evolution of blood feeding [15, 41-43, 92, 94-100]. Many of the salivary protein sequences are coded by genes related to intrinsic functions of the cell (housekeeping genes). However, the large number of salivary proteins is secreted during plant or blood feeding. Finally, a little number has no similarities to sequences deposited in databases, representing unknown and novel sequences $[41,94,101]$. This emphasizes how much still need to be learned concerning the biological functions of salivary proteins in blood feeding, pathogen transmission and manipulation of host responses.

The analysis of the adult Anopheles sialome has shown that secreted proteins and/or peptides (secretome) can be ubiquitous or specific to arthropod classes, orders, families, genus or species 


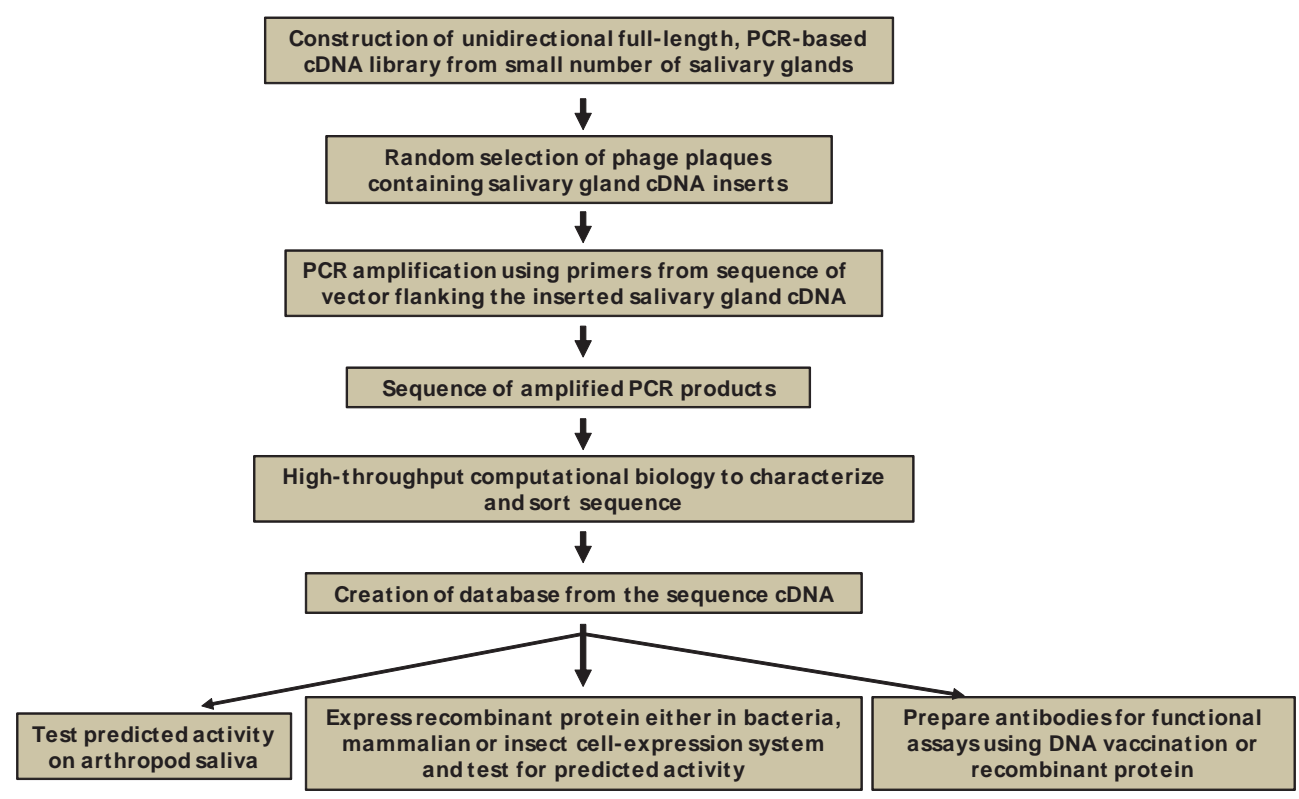

Figure 7. Current high-throughput strategies used for the isolation and characterisation of salivary cDNA and proteins from disease vectors [90].

[44, 101, 102]. In An. gambiae salivary gland females over 70 putative secreted salivary proteins have been identified [94].

\subsubsection{Ubiquitous salivary proteins}

AG5 family proteins are found in the salivary glands of many blood-sucking insects and ticks $[102,103]$. In An. gambiae, four proteins belonging to this family were identified, but only one (putative gVAG protein precursor) was coding for transcripts enriched in the adult female SGs [94]. A precursor of gVAG protein was also described in An. funestus (84\% sequence identity) and An. stephensi (85\% sequence identity) sialome [95, 100]. The function of any AG5 protein in the saliva of any blood-sucking arthropod is still unknown.

Enzymes such as maltase, apyrase, $5^{\prime}$ nucleotidase, and adenosine deaminase, are also secreted during the bite of many blood-sucking arthropods, including Anopheles mosquitoes [95]. They generally assist in sugar feeding (maltase) or in degradation of purinergic mediators of platelet aggregation (apyrase, $5^{\prime}$ nucleotidases) and inflammation (adenosine deaminase).

\subsubsection{Salivary proteins found exclusively in Diptera}

D7 family proteins are specific to SGs of blood-sucking Nematocera, including mosquitoes and sand flies [104, 105]. They are highly represented in the sialome of Anopheles mosquitoes in short and long forms $[95,96,101,104,105]$. An. funestus D7 proteins vary between $64 \%$ and 
$75 \%$ identity with their An. gambiae closest match [105]. D7 proteins could act as anti-hemostatic factors by trapping agonists of hemostasis [44, 47]. However, further investigations are needed to clearly describe their function.

Other Diptera-specific protein families or peptides have also been described in the sialome of blood-feeding mosquitoes [95]. However their function is still unknown, even if some were known to play a role in antimicrobial property of mosquito saliva.

\subsubsection{Protein families found exclusively in mosquitoes}

The 30-kDa antigen family found exclusively in the SGs of adult female mosquitoes has been found in both culicine and anopheline mosquitoes [95, 100, 101, 106-108]. Only one gene enriched in SGs of adult females is known in An. gambiae. The An. funestus homologue is also abundantly expressed and shares $63 \%$ identity with the An. gambiae orthologue. The function of this protein family is still unknown [95].

The gSG (An. gambiae Salivary Gland)-5 family was first discovered in the SGs of An. gambiae and shown to be exclusively expressed in the adult female [94, 109]. This protein shows a high similarity to Aedes and Culex proteins [101]. Transcripts coding for this family were found in the sialotranscriptome of An. darlingi with $46 \%$ identical to the An. gambiae orthologue and only $26 \%$ and $23 \%$ identical to the culicine proteins [101]. The function of this mosquito-specific protein remains unknown, but its tissue- and sex-specific expression profile suggests it is possibly related to blood feeding.

The gSG8 family is highly divergent with members only found in An. gambiae and Ae. aegypti. In An. gambiae, this protein is specifically expressed in female SGs [109], suggesting a likely role in blood feeding.

Various types of mucins have been described in the saliva of adult mosquitoes and may function/act as a lubricant of their mouthparts [15, 41, 94, 102]. Three mucins encoding transcripts have been identified in the An. gambiae larval SG [110], suggesting the importance of mucins at multiple developmental stages. Mucins may also play a crucial role in Anopheles salivary gland invasion by $P$. berghei sporozoites [111]. Several protein families are also represented in this group, including gSG-3, gSG-10, and 13.5-kDa families [101]. These families were also found abundantly expressed in the sialotranscriptome of An. gambiae adult male [112], indicating their function is not related specifically to blood feeding.

\subsubsection{Protein families found exclusively in Anophelines}

Anophelin was described as a short acidic peptide with strong thrombin inhibitory activity in An. albimanus [46]. An. funestus anophelin is 59\% identical to the An. gambiae orthologue [95], and An. darlingi anophelin is $86 \%$ identical to An. albimanus [101].

The 8.2-kDa family is represented in several Anopheles species. In An. funestus the peptide have $42 \%$ identity with the $8.2-\mathrm{kDa}$ salivary peptide of $A n$. stephensi and similar proteins from $A n$. gambiae and An. darlingi [95]. In An. gambiae, this peptide was found enriched in adult female SGs, suggesting a role in blood feeding. 
The 6.2-kDa family was first described in a sialotranscriptome of An. gambiae [94], where it was found enriched in adult female SGs compared to other tissues. The An. funestus member of this family is $61 \%$ identical to the An. gambiae [95], and 53\% to an An. darlingi [101] homologues.

The SG-1 family proteins appear to be exclusively expressed in the female SGs of Anopheles mosquitoes and not observed in other tissues [94, 101]. However, their function remains to be determined.

The SG-2 family proteins were identified from An. gambiae saliva and shown to be expressed in female SGs and adult males but not in other tissues [113]. Related, but very divergent, sequences were obtained from salivary transcriptomes of other anopheline species [95, 101]. Because this protein family is expressed in both male and female An. gambiae, and due to its relatively small size, it may display antimicrobial function [101].

The hyp 8.2 and hyp 6.2 proteins are similarly enriched in An. gambiae adult female SGs [94]. An. stephensi and An. funestus also have members of these protein families.

The SG-7/Anophensin family is also unique to anophelines. In An. gambiae, it is highly enriched in female SGs [94]. More recently, the An. stephensi homologue was determined to inhibit kallikrein and production of bradykinin, a pain-producing substance [114]. Four putative alleles representing the homologue(s) of gSG7 in An. darlingi were identified. These An. darlingi transcripts have no more than $45 \%$ identity to the An. gambiae gSG7 and An. stephensi anophensin [101].

The SG6 protein is a small protein first described in An. gambiae [109] and a unique sequence codes for a mature peptide/protein of $\sim 10 \mathrm{kDa}$ (116 amino-acids) with ten cysteine residues making probably five disulphide bonds. A homologue was later found in the sialotranscriptome of An. stephensi [100] and An. funestus [95]. An. funestus SG6/fSG6 (f for funestus) has 81\% and $76 \%$ identities with $A n$. stephensi and $A n$. gambiae polypeptides, respectively. It is not found in the transcriptomes of the Culicinae subfamily members analyzed so far, i.e. C. pipiens quinquefasciatus, Ae. aegypti and Ae. albopictus [108, 115, 116]. In An. gambiae, the transcript coding for gSG6 ( $\mathrm{g}$ for gambiae) was found to be 16 times more expressed in SGs of adult females than in males [94]. The gSG6 protein plays some essential blood feeding role and was recruited in the anopheline subfamily most probably after the separation of the lineage which gave origin to Cellia and Anopheles subgenera [99]. The gSG6 protein, because immunogenic, can be therefore a reliable indicator of human exposure specific to Anopheles mosquito bites [99], vectors of malaria.

\subsection{Specific salivary biomarker of exposure to Anopheles bites: The gSG6-P1 peptide candidate}

The SG6 salivary protein has been reported to be immunogenic in travelers exposed for short periods to Anopheles bites [21], and in Senegalese children living in a malaria endemic area by an immuno-proteomic, coupling 2D immunoblot and mass spectrometry [117], and by an ELISA [26] approaches. Recently, its immunogenicity has been confirmed in individuals from a malaria hyperendemic area of Burkina Faso [118,119], by using a recombinant form expressed as purified N-terminal His-tagged recombinant protein in the E. coli vector pET28b(+) (Novagen) $[99,119]$. 

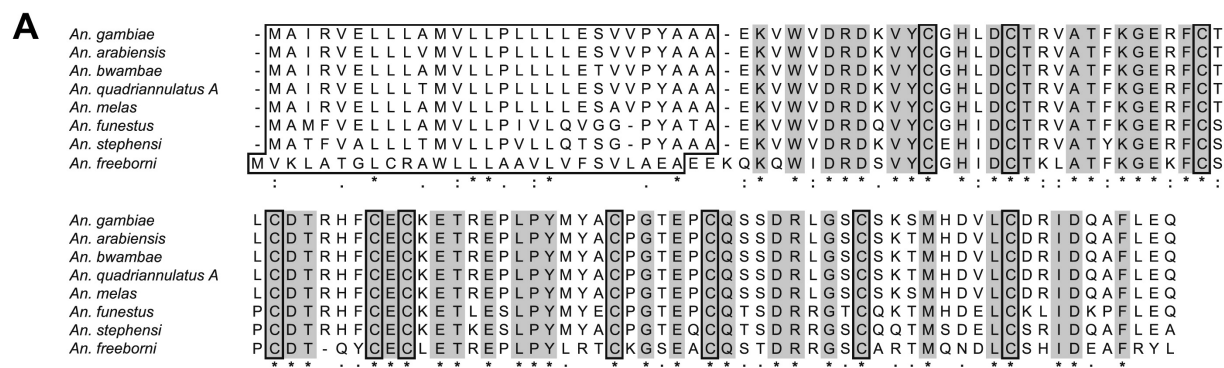

LCD TRHFCECKETREPLPYMYACPGTEPCQSSDR L GSCSKSMHDVLCDR I DQAFLEQ

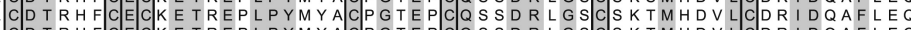
CDTRHFCE C K E TREPLPYMYACPGTEPCQSSDRLGSCSKTMHDVLCDR I DQAFLE CDTRHFCECKE TREPLPYMYACPGTEP CQSSDRLGSCSKTMHDVLCDR D D A A LE Q CDTRHFCECKETREPLPYMYACPGTEPCQSSDRLGSCSKSMHDVLCDRIDQAFLEQ CDTRHFCECKE TLESLPYMYECPGTEPCQTSDRRGTCQKTMHDELCKLIDKPFLE PCDTRHFCECKETKESLPYMYACPGTEQCQTSDRRGSCQQTMSDELCSRIDQAFLEA

B

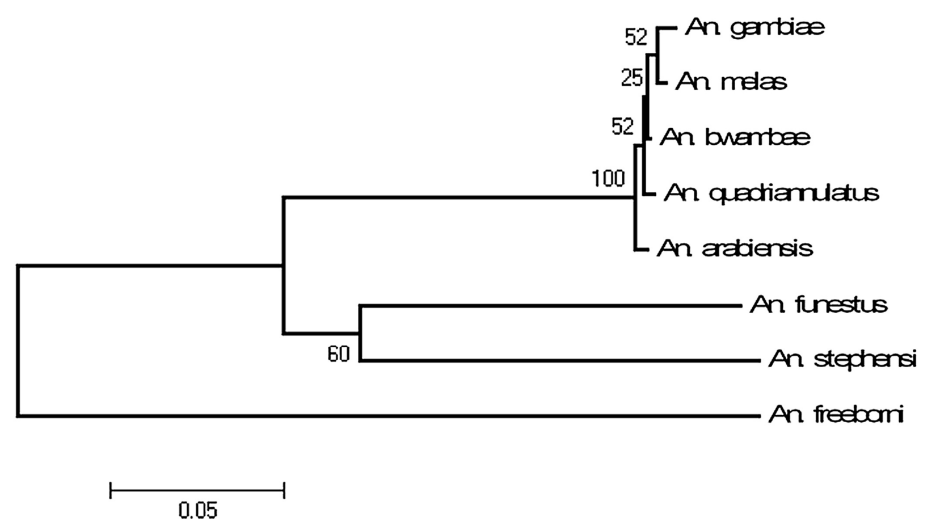

Figure 8. Sequences of the anopheline gSG6 proteins [99]. (A) Clustal alignment of anopheline gSG6 proteins. Signal peptides and conserved Cysteines are boxed. Conserved sites are shaded. (B) Phylogenetic tree (NJ algorithm, bootstrapped 10,000 times) constructed from the alignment of the nucleotide sequence encoding the mature gSG6 polypeptides.

In particular, increased anti-gSG6 IgG levels were observed in exposed individuals during the malaria transmission/rainy season [119]. In addition, anti-gSG6 IgG response appeared to be a reliable serological indicator of exposure to bites of the main African malaria vectors(An.gambiae, An. arabiensis and An. funestus) in the same area [119]. However, gSG6 recombinant protein has been described to relatively generate a high background in control sera from individuals not exposed to Anopheles bites, and considerable variations in specific Ab response between children supposed to be similarly exposed to Anopheles bites [26]. Therefore, with the objective of optimizing Anopheles specificity and reproducibility of the immunological assay, a peptide design approach was undertaken using bioinformatic tools [26].

\subsubsection{Identification and sequence of gSG6-P1 peptide}

Several algorithms were employed for prediction of potential immunogenic sites of the gSG6 protein by using bioinformatics. The prediction of immunogenicity was based on the determination of physico-chemical properties of the amino-acid (AA) sequences with BcePred and FIMM databases and on the identification of MHC class 2 binding regions using the ProPred-2 online service. This led to define five gSG6 peptides (gSG6-P1 to gSG6-P5) of 20 to 27 AA 
residues in length (Fig. 9), overlapping by at least 3 residues and spanning the entire sequence of the mature gSG6 protein. Both predictive methods for putative linear B-cell epitopes (FIMM and BcePred) assigned the highest immunogenicity to gSG6-P1, gSG6-P2, gSG6-P3, and then gSG6-P4.

\section{MAIRVELLLAMVLLPLLLLESVVPHAAAEKVWVDRDNVY}

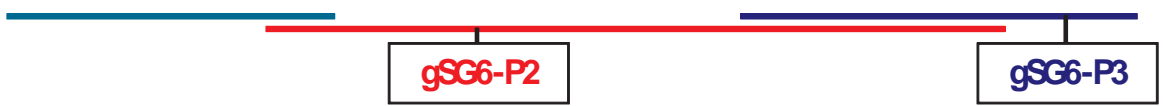

ACPGTEPCQSSDRLGSCSKSMHDVLCDRIDQAFLEQ

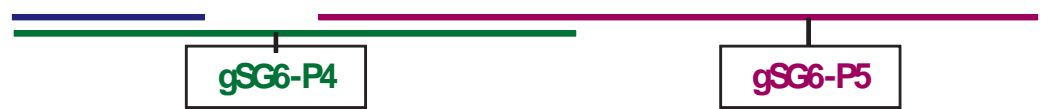

Figure 9. Amino-acid sequence of gSG6 Peptides. Amino-acid sequence of the SG6 protein of Anopheles gambiae (gi: 13537666) is presented and sequences of the selected peptides, gSG6-P1 to gSG6-P5, are underlined. Signal peptide (SP) sequence is indicating by dotted underline [26].

Similarities were also searched using the Blast family programs, including both the genome/EST libraries of other vector arthropods available in Vectorbase and of pathogens/ organisms in non-redundant GenBank CDS databases. No relevant identity was found with proteins of other blood-sucking arthropods. Indeed, the longest perfect match was 6 AAs between a putative protein from Pediculus humanus and gSG6-P2 and gSG6-P3 peptides. In the case of gSG6-P1, the best match was 4 AAs in length with Culex pipiens quinquefasciatus salivary adenosine deaminase. Moreover, no relevant similarity was found with sequences from pathogens or other organisms. The highest hits of gSG6-P1 were with the cyanobacterium Microcystis aeruginosa (3 AAs) and with Ostreococcus OsV5 virus (4 AAs). Altogether, this analysis confirmed the bona fide high specificity of the five selected gSG6 peptides for the Anopheles species. Peptides were then synthesized.

\subsubsection{Antigenicity of gSG6 peptides}

IgG $\mathrm{Ab}$ responses to the five gSG6 peptides were evaluated by ELISA in a randomly selected subsample of children $(\mathrm{n}<30)$ living in a rural area of Senegal. All peptides were immunogenic, but the intensity of the IgG level was clearly peptide-dependent; weak immunogenicity was observed for gSG6-P3, gSG6-P4 and gSG6-P5, whereas gSG6-P1 and gSG6-P2 appeared highly immunogenic (Fig. 10). 


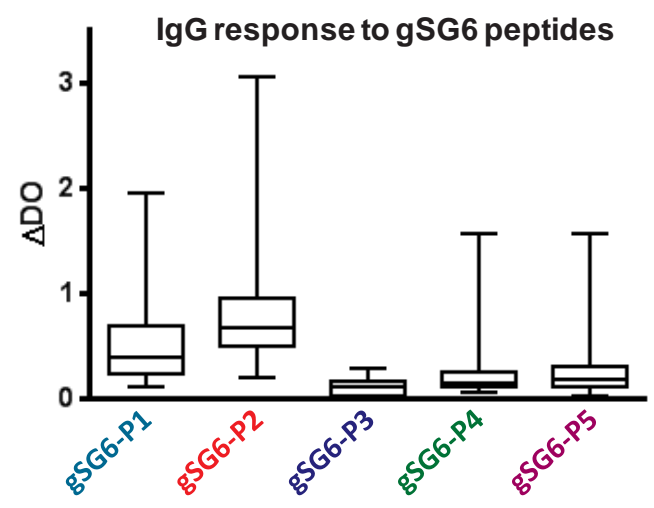

Figure 10. IgG antibody response according to gSG6 peptides [26]. For each peptide, the IgG Ab level was evaluated in a subsample of exposed children. Results at the peak of the season of Anopheles exposure are reported according to gSG6 peptides. Results are presented by box plot graph where lines of the boxes represent the 75th percentile, median and 25 th percentile of individual average $\triangle O D$ values; whiskers represent the lower and upper adjacent values.

\subsubsection{Validation as a biomarker of exposure in several epidemiological settings}

The specific IgG level to the two most antigenic gSG6 peptides (gSG6-P1 et gSG6-P2) was then evaluated according to the level of exposure (estimated by entomological data) in a larger sample $(\mathrm{n}=241)$ of children living in a malaria seasonal area [26]. A positive trend was found for both peptides, but only significant for gSG6-P1 (Figure 11). Altogether, these results indicated that only the IgG response to gSG6-P1 is suitable to be a pertinent biomarker of exposure to Anopheles bites and thus to risk of malaria.
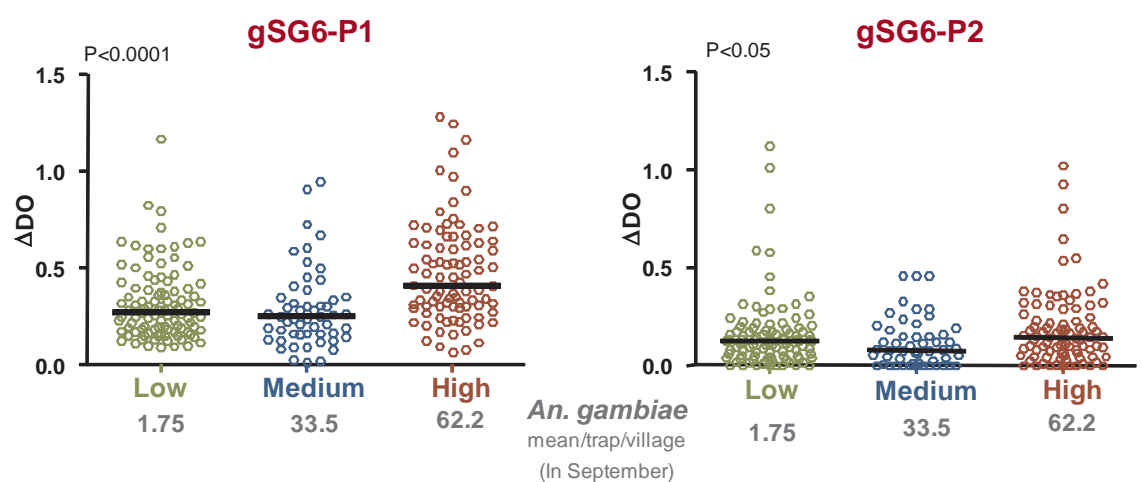

Figure 11. IgG response to gSG6-P1 and gSG6-P2 according to intensity of exposure to Anopheles gambiae bites [26]. Individual $\triangle O D$ (Optical Density) values in September (peak of the season of Anopheles exposure) are shown for the three different exposure groups. Results are presented for the same children ( $n=241)$ for gSG6-P1 (A) and gSG6-P2 (B). Exposure groups were defined by entomological data. Bars indicate median value for each exposure group. Statistical significance between the 3 groups is indicated (non-parametric Mann-Whitney U-test). 
Therefore, the gSG6-P1 was selected as the most pertinent candidate as marker of exposure. Indeed, this peptide appeared to satisfy several requirements that an exposure biomarker should fulfill. First, it thus far appears to be specific to Anopheles genus and therefore, no relevant cross-reactivity phenomena with epitopes from other proteins of arthropods or pathogens would be expected. Second, because it is of a synthetic nature, it guarantees high reproducibility of the immunological assay. Third, it elicits a specific Ab response which correlates well with the level of exposure to An. gambiae bites.

\subsubsection{Biomarker of Anopheles vector bites}

As previously suggested, anti-gSG6-P1 IgG response was described as a biomarker of An. gambiae bites in children living in Senegalese villages where malaria transmission seasonally and moderately occurred [26]. In the same area, a specific IgG response to the peptide has been detected in $36 \%$ of children living in villages where very few An. gambiae, or none, were collected by classical entomological methods [28]. This deals with a high sensitivity and specificity of the gSG6-P1 epitope(s) after a low immunological boost induced by weak bites exposure. This result points to the potential use of such serological tool as an epidemiological biomarker of $A n$. gambiae bites in very low exposure areas, where the sensitivity of current entomological methods of malaria risk assessment is weak.

One study aimed to evaluate the risk of malaria transmission in children and adults living in urban area of Senegal (Dakar region) by using the gSG6-P1 peptide biomarker. Results showed considerable individual variations in anti-gSG6-P1 IgG levels between and within districts, in spite of a context of a global low Anopheles exposure level and malaria transmission [27]. Despite this individual heterogeneity, the median level of specific IgG and the percentage of immune responders differed significantly between districts. In addition, a positive association was observed between the exposure levels to An. gambiae bites, estimated by classical entomological methods, and the median IgG levels or the percentage of immune responders reflecting the real contact between human populations and Anopheles mosquitoes [27]. Differences in exposure levels to An. gambiae bites could then partly explain district and/or group-variations in anti-gSG6-P1 IgG Ab response as previously described in a low-exposure rural area of Senegal [28]. Interestingly, in urban Dakar area, immunological parameters seemed to better discriminate the Anopheles exposure level between different groups compared to referent entomological data. Moreover, in this study, some discrepancies were observed in the correlation between immunological parameters and the exposure level to An. gambiae bites assessed by entomological data in districts. This suggests the main role of the human behavior influencing the contact with vectors. A differential use of Vector Control Measures (ITNs, sprays, curtains) can for example drastically reduce human-vector contact. Many household characteristics (height, type, use of air conditioning, well-closed windows), which can differ between districts, could also be crucial factors. Importantly, the effect of these factors may be not taken into account by assessing the mosquito exposure level and malaria risk with classical entomological tools. This strengthens the usefulness of such biomarker as an alternative tool in the evaluation of exposure levels to Anopheles bites, especially in low/very low exposure, where current entomological methods can give inaccurate estimations of the human-mosquito contact [27]. 
In a population from a malaria hyperendemic area of Burkina Faso, the use of gSG6 recombinant protein as reliable indicator of exposure to the 3 main African malaria vectors (An. gambiae s.s., An. arabiensis and An. funestus) has been suggested [119]. This probably could be relied to a wide cross-reactivity between SG6 sequences of principal Anopheles vectors, which highly share identical epitopes between species. Moreover, the gSG6-P1 peptide has been used to accurately evaluate the exposure level to An. funestus bites in a rural area in Senegal [29]. Indeed, two-thirds of 2-9 years old children from this area developed an IgG response to gSG6$\mathrm{P} 1$, in an area where An. funestus only was reported. In addition, IgG response increased during the An. funestus exposure season, and a positive association was observed with the level of exposure to An. funestus bites [29]. This result deals with the cross-reactivity between $A n$. gambiae gSG6-P1 and An. funestus fSG6-P1 sequences which share a high level of identity. Indeed, these sequences differ only by the substitution of two AAs: asparagine by glutamine (position 9) and leucine by isoleucine (position 15) (Fig. 12).

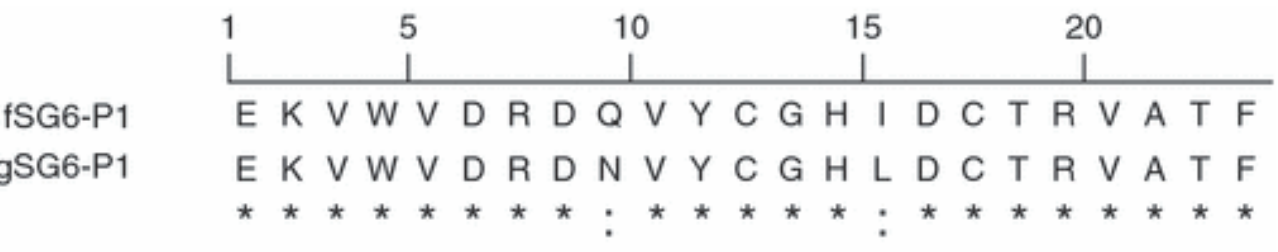

Figure 12. Sequences of the SG6-P1 salivary peptide [29]. Sequences are shown for An. funestus (fSG6-P1), for An. gambiae (gSG6-P1). Identities are marked with '*' and strong AA conservations with ':'.

AAs from fSG6-P1 are close in terms of polarity and charge to those from An. gambiae gSG6P1. The main consequence is that individuals exposed to An. funestus bites can sufficiently develop a specific Ab response against gSG6-P1 An. gambiae antigen. This observation, in conjunction with present results, suggests that these substitutions do not alter the synthesis and the recognition of specific $\mathrm{Ab}$ because epitope appears to be conserved.

All mentioned studies were conducted on subjects older than 1 year. However, to be more relevant in epidemiological surveys and studies on malaria, such biomarker tool must pertinently be applicable to all human age-classes, including newborns and young infants $(<1$ year old) who can be also bitten by Anopheles and at high risk of malaria transmission [120]. In this way, a recent study has indicated that human Ab responses to gSG6-P1 biomarker help to assess Anopheles exposure level and the risk of malaria in younger than 1 year old infants living in moderate to high transmission area of Benin (Drame et al., submitted).

Indeed, the presence of anti-gSG6-P1 IgG and IgM in the blood of respectively 93.28 and $41.79 \%$ of 3-months old infants (the majority of infants) and their gradual increasing levels until 12 months (Fig. 13), whatever the Anopheles exposure level or the season. These observations are consistent with the development and maturation patterns of the newborn immune system during the first months of life. Indeed, the immature human immune system completes its maturation during infancy following exposition to antigens. Therefore, newborns are naive and increasingly susceptible to infectious agents; their immune system is not or insufficiently 

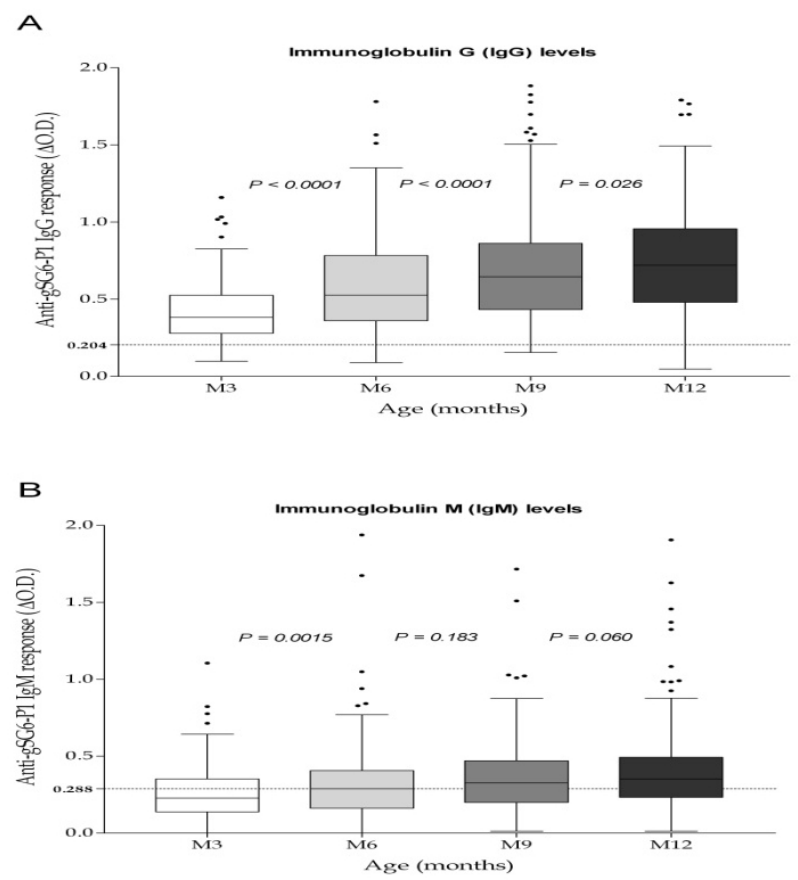

Figure 13. IgG and IgM responses to Anopheles gSG6-P1 salivary peptide in the first year-life. Individual IgG (A) and IgM (B) responses to the Anopheles gSG6-P1 are represented for infants in months 3 (white), 6 (light-gray), 9 (darkgray) and 12 (black box) after their birth. Horizontal lines in the boxes indicate medians of the individual data. Horizontal black dotted lines represent the cut-off of $\operatorname{lgG}(0.204)$ and $\lg M(0.288)$ responder. Statistical significant differences between all age groups (multivariate linear mixed model analysis) are indicated.

stimulated by antigens. In endemic malaria transmission area, they are progressively exposed to salivary antigens of Anopheles [121], probably explaining the progressive increase of antigSG6-P1 IgG and IgM from 3 to 12 months-old. Individual or population factors and behaviors enhancing the level of the human-Anopheles contact with age can play a crucial role on accelerating this gradual acquisition [122, 123].

\subsubsection{Factors of variation of antibody response to gSG6-P1 and their consequences}

Specific gSG6-P1 Ab responses can be influenced by several determinant factors in their variations between individuals, districts, villages, regions... Therefore, identifying effects of human intrinsic (gender, age...) and extrinsic (period of sampling, use of vector control measure...) factors will be useful to the application of the gSG6-P1 biomarker in epidemiological studies or monitoring, evaluation and surveillance of risk of malaria programmes.

\section{Effect of age}

Studies have globally reported an increasing anti-gSG6-P1 Ab level according to individual age. In a moderate transmission semi-urban area in Angola, the lowest and highest specific 
IgG levels have been described in young children ( $0-7$ years old) and in teenagers/ adults (>14 years old) respectively [30]. In a low malaria transmission urban area (Dakar region) in Senegal, specific IgG levels were significantly higher in adults (>18 years old) compared to 6-10 years old children and in this latter group compared to those aged from 2 to 5 years [27] [124]. In Tori Bossito, moderate-high rural transmission area of Benin, both anti-gSG6-P1 IgG and IgM levels were low at 3 months of age and gradually increased until 12 months after birth (Drame et al., submitted). The increase of specific IgG response with age is consistent with the gradual acquired immunity against Anopheles mosquito saliva [30] following the development of individual factors and behaviors enhancing the probability of human-vector contact [122, 123]. However, few data have reported a decrease of IgG levels to gSG6-P1 peptide [28] or to SG6 protein [118] with age. In particular, in Senegalese children (0 to 60 months old), the highest specific IgG levels were reported in the youngest children in spite of a probable very weak exposure to An. gambiae [30]. It can be explained by a passive IgG transfer from mother to child during pregnancy or breastfeeding as recently reported in young infants from Benin (Drame et al., submitted). This represents a way of overestimation of the assessment of humanAnopheles contact level and the risk of malaria in young infants by using anti-gSG6-P1 IgG Ab. Therefore, the evaluation of specific IgM Ab levels could be a relevant solution to bias in IgG measurements. Indeed, IgM Ab, in a form of polymers (usually pentamers) in the human organism, could not cross the maternal-foetal barrier [125] and are the first Ab to appear in response to initial or primary exposure to antigen [126]. Interestingly, in Tori Bossito, specific IgM levels seemed to be a serological marker only during the first 6-months of exposure. In infants older to 6 months, the assessment of gSG6-P1-specific IgG showed a more pertinent evaluation of exposure level.

\section{Effect of sex}

Some studies have reported higher levels of anti-gSG6-P1 in female individuals (children and women) compared to males (children and men) [27, 30] ([124]; Drame et al., submitted). However, this difference was not significant, suggesting that it might be only physiological.

\section{The season of Anopheles exposure}

The season of individual sampling may be also a factor of confusion in the use gSG6-P1 biomarker in epidemiological studies on malaria risk assessment or control. Indeed, significant seasonally variations in anti-gSG6-P1 IgG or/and IgM levels have been reported in studies conducted in newborns, children or/and adults from endemic malaria areas in Senegal [27-29, 124], Angola [30] and Benin (Drame et al., submitted). In Senegal, in particular, specific gSG6-P1 in urban children and adults steadily waned from the beginning (October) to the end (December) of the study, due to an important drop in human exposure level to An. gambiae s. l. bites from the end of rainfalls (October) to the beginning of the dry season (December) [127, 128].

One direct application of a salivary biomarker of exposure could serve in the elaboration of maps representing the risk of exposure to Anopheles bites. Such immuno-epidemiological marker might represent a quantitative tool applied to field conditions and a complementary tool to those currently available, such as entomological, ecological and environmental data [59, 129]. It could represent a geographic indicator of the risks of malaria transmission and thus a 
useful tool for predicting malaria morbidity risk as previously described [22]. Furthermore, it may represent a powerful tool for evaluation of vector control strategies (impregnated bednet, intradomiciliary aspersion, etc.) and could here constitute a direct criterion for effectiveness and appropriate use (malaria control program) [84].

\subsubsection{Indicator of malaria vector control effectiveness}

\section{Long and short-term evaluation of ITN efficacy}

A longitudinal study associating parasitological, entomological and immunological assessments of the efficacy of ITN-based strategies using the gSG6-P1 biomarker has been conducted in a malaria-endemic area in Angola. Human IgG responses to gSG6-P1 peptide were evaluated in 105 individuals (adults and children) before and after the introduction of ITNs and compared to entomo-parasitological data. A significant decrease of anti-gSG6-P1 IgG response was observed just after the effective use of ITNs (Fig. 14). The drop in gSG6-P1 IgG levels was associated with a considerable decrease of $P$. falciparum parasitaemia, the current WHO criterion for vector control efficacy [130]. It was particularly marked in April-August 2006, corresponding to the season peak of An. gambiae exposure. Interestingly, the entomological data indicated that this season-dependent peak was of similar intensity before (2005) and after (2006) ITN use, suggesting ITN installation had no impact on An. gambiae density, probably because of the low percentage of the overall human population covered in the studied area [131]. This study indicated also that the drop of anti-gSG6-P1 IgG response was associated with correct ITN use and not due to low Anopheles density. In addition, this was observed in all age groups studied ( $<7$ years, $7-14$ years, and $>14$ years), suggesting that this biomarker is relevant for ITN evaluation in all age groups. This rapid decrease after correct ITN usage appears to be a special property of anti-gSG6-P1 IgG which is short-lived (4-6 weeks) in the absence of ongoing antigenic stimulation, at/for all age classes.

The response does not seem to build up but wanes rapidly, when exposure failed. This property represents a major strength when using such salivary biomarker of exposure for evaluating the efficacy of vector control. In addition, using a response threshold $(\triangle \mathrm{OD}=0.204)$ combined with $\Delta \mathrm{OD}_{\mathrm{ITNs}}$ - the difference between April (after ITNs) and January 2006 (before) - makes possible the use of this operational biomarker at individual level (Fig. 15). The threshold response (TR) represents the non-specific background IgG response (the cut-off of immune response) and was calculated in non-Anopheles exposed individuals $(\mathrm{n}=14$ - neg; North of France) by using this formula: $\mathrm{TR}=$ mean $\left(\Delta \mathrm{DO}_{\text {neg }}\right)+3 \mathrm{SD}=0.204$. An exposed individual was then classified as an immune responder if its $\Delta \mathrm{OD}>0.204$. If the $\Delta \mathrm{OD}_{\mathrm{ITNs}}$ value is comprised between -0.204 and +0.204 , no clear difference in exposure level to Anopheles bites can be defined.

In contrast, if the individual $\Delta \mathrm{OD}_{\mathrm{ITNS}}$ value $<-0.204$, it could be concluded with a high level of confidence that this individual is benefiting from ITN installation. The $\Delta \mathrm{OD}_{\text {ITNs }}$ parameter could therefore provide a measure of ITN efficacy at the individual level. An individual biomarker would also be relevant at the large-scale operational studies or surveillance in the field, e.g. in National Malaria Control Programs (NMCP). In addition, the high sensitivity and specificity of the gSG6-P1 Ab response make it ideal for the evaluation of low-level ex- 

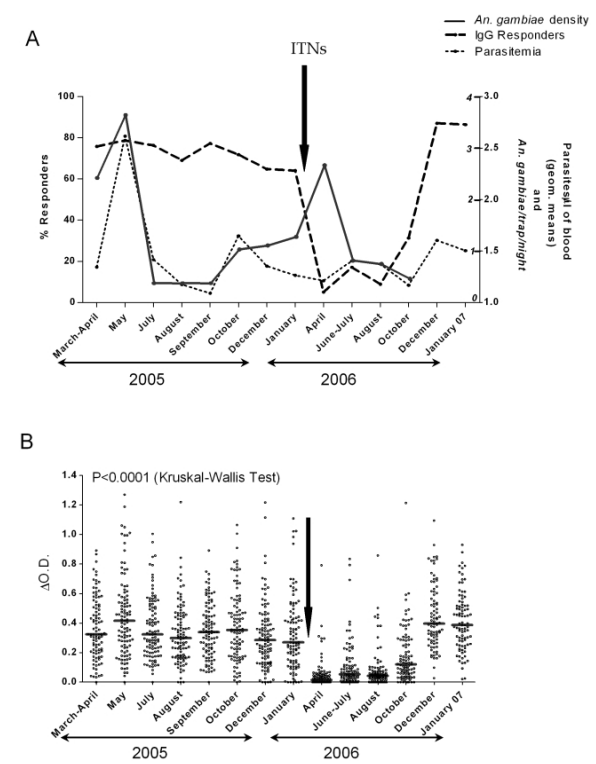

Figure 14. IgG Ab responses to gSG6-P1 before and after ITN use [30]. The percentage (\%) of anti-gSG6-P1 IgG immune responders (thick-dotted line) in the "immunological" sub-population ( $n=105)$, before (2005) and after (2006 and January 2007) the installation of ITNs (A). These results are presented together with the intensity of $P$. falciparum infection (mean parasitaemia - fine-dotted line) measured in the same population and the mean of number of An. gambiae (solid line) in the studied area (A). Entomological data were not available in December 2006 and January 2007 (the last two months of the study). Arrows indicate the installation of Insecticide Treated Nets (ITNs) in February 2006. Individual anti-gSG6-P1 IgG levels ( $\triangle O D$ ) are presented before (2005) and after (2006) the installation of ITNs (B). Bars indicate the median value for each studied month. Statistically significant differences between months are indicated.

posure to Anopheles bites [27, 28], even when exposure or transmission is curtailed by NMCP efforts. Taken together, the estimation of human IgG responses to Anopheles gSG6-P1 could provide a reliable indicator for evaluating the efficacy of ITN-based strategies against malaria vectors, at individual and population levels, even after vector control generating particular low exposure/transmission contexts. This salivary biomarker is a relevant tool for the evaluation of short-term efficacy as well as longer-term monitoring of malaria VCMs.

\section{Evaluation of effectiveness of diverse vector control measures}

A recent cross-sectional study conducted from October to December 2008 on 2,774 residents (children and adults) of 45 districts of urban Dakar (Senegal) has validated IgG responses to gSG6-P1 as an epidemiological indicator evaluating the effectiveness of a range of VCMs. Indeed, in this area, IgG levels to gSG6-P1 as well as the use of diverse malaria VCMs (ITNs, mosquito coils, spray bombs, ventilation and/or incense) highly varied between districts [124]. This difference of use suggests some socio-economical and cultural discrepancies between householders as described in large cities of Ivory Coast [132] and Tanzania [123]. At the district level, specific IgG levels significantly decreased with VCM use in children as well as in adults. 
A

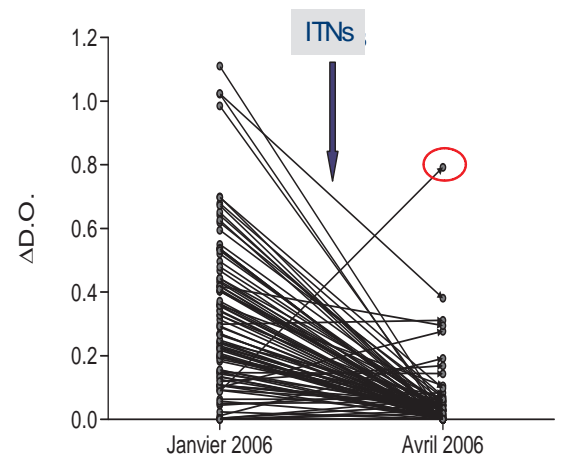

B

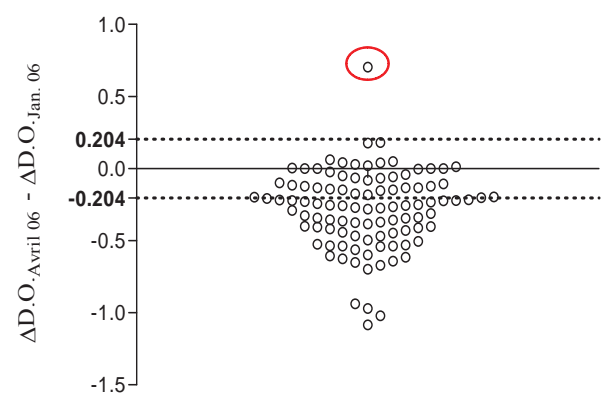

Figure 15. IgG response to gSG6-P1 as biomarker for short-term ITN efficacy. Changes in individual IgG levels ( $\triangle \mathrm{OD})$ are presented between "just before" (January 2006) and "just after" (April 2006) ITN introduction ( $n=105$; children and adults) (A). The arrow indicates the installation of Insecticide Treated Nets (ITNs) in February 2006. Individual IgG level changes from January (before) to April are presented (B) by individual $\triangle O D_{\text {ITNs, }}$ value $\left(\triangle O D_{\text {ITNs }=} \Delta O D_{\text {April06, }}\right.$ $\left.\triangle O D_{\text {Januaryo6 }}\right)$. The threshold of specific IgG responders $(T R=0.204)$ is indicated (dotted line). Significant positive $(\triangle O D>0.204)$ or negative $(\triangle O D<-0.204)$ changes are therefore individually presented.

Among used VCM, ITNs, the $1^{\text {st }}$ chosen preventive method ( $43.35 \%$ rate of use), by reducing drastically the human-Anopheles contact level and specific IgG levels in children as well as in adults, were by far the most efficient whatever age, period of sampling or the exposure level to mosquito bites. Spray bombs were secondarily associated to a decrease of specific IgG level, due certainly to their power and fast knock-down action. But, their effects can be limited by the non-persistence of used products and some socio-economic considerations [133]. In addition, they only have been recently adopted and are more expensive in the majority of subSaharan Africa cities [133], explaining their less frequent use (9.57\% rate of use) in the Dakar area. The non-effect of mosquito coil use is surprising, regardless to their well-adoption by residents (36.68\% of rate of use), but it can be explained by their power deterrent effect which tends to push Anopheles vectors outside where they can remain active [133]. However, the protection ensured by ITN use seemed to be insufficient because anti-gSG6-P1 IgG levels in ITN users were specifically high in some periods of fairly high exposure to Anopheles bites. Changes in An. arabiensis behaviour, the major malaria vector in the area, can also explain this lack of protection. It can bite outside the rooms/ habitations with a maximal activity around $10.00 \mathrm{pm}$, when people are not in bed and ITNs not hanged [123]. Therefore, ITNs must be associated to a complementary VCM for an effective protection against Anopheles bites.

Taken together, these results suggest that the assessment of human IgG responses to Anopheles gSG6-P1 salivary peptide can provide a reliable evaluation of the effectiveness of malaria vector control in urban settings of Dakar whatever the age, sex, level of exposure to bites or period of malaria transmission. Therefore, this salivary biomarker can be used to compare the effectiveness of different anti-malaria vector strategies in order to identify the most suitable for a given area. 


\section{Comparing effectiveness of combined or not vector control measures}

In parallel to an entomological and parasitological evaluation, IgG responses to gSG6-P1 were also used to assess, in a randomized controlled trial in 28 villages in southern Benin, four malaria vector control interventions: Long-Lasting Insecticide-treated Net (LLIN) targeted coverage to pregnant women and children younger than 6 years (TLLIN, reference group), LLIN universal coverage of all sleeping units (ULLIN), TLLIN plus full coverage of carbamateindoor residual spraying (IRS) applied every 8 months (TLLIN+IRS), and ULLIN plus full coverage of carbamate-treated plastic sheeting (CTPS) lined up to the upper part of the household walls (ULLIN+CTPS). Results from this study have shown that specific IgG levels were similar in the 4 groups before intervention and only significantly lower in the ULLIN group compared to the others after intervention. In contrast to immunological data, clinical incidence density of malaria, the prevalence and parasite density of asymptomatic infections, and the density and aggressiveness of Anopheles mosquitoes, were not significantly different between the four groups before as well as after interventions [134]. These findings mean that LLIN used along by all the population of a given area may be more suitable in reducing the contact between human populations and the Anopheles vectors, even if any effect on malaria morbidity, infection, and transmission was not observed. Therefore, combining anti-vector tools do not undeniably reduce individual exposure to malaria vectors, even if significant effect on reducing more rapidly malaria transmission and burden has been reported [135]. These findings confirm that anti-vector saliva $\mathrm{Ab}$ response as a biomarker of exposure is also important for NMCPs and should help the design of more cost-effective strategies for malaria control and elimination.

\subsubsection{Importance to develop a specific biomarker of infecting Anopheles bites}

Recent data have shown that the use of the gSG6-P1 biomarker for the assessment of the differential risk of the disease transmission may have some limitations in high exposure areas (Drame et al., submitted). Indeed, the gSG6-P1 assesses the exposure level to both infective and not infective Anopheles bites. In malaria hyperendemic areas, resident people are highly exposed to mainly not infective bites and present almost all Ab specific to gSG6-P1 levels relatively high. Therefore it should be relevant to develop a biomarker of exposure specific to infective bites in order to assess the human risk of malaria transmission in such contexts. Such epidemiological parameter would be important to define in the context of malaria control. The transmission depends on the density of competent Anopheles, of their Plasmodium infective rate and of the intensity of human-vector contact. In addition, current methods to measure the intensity of malaria transmission show several limitations, especially in low transmission areas. The EIR (entomological inoculation rate) is a commonly used metric rate that estimates the number of bites by infectious mosquitoes per person per unit time. It is the product of the "human biting rate" - the number of bites per person per day by vector mosquitoes - and the fraction of vector mosquitoes that are infectious (the "sporozoite rate"). The classical method to estimate the density of sporozoites in mosquitoes is the dissection of salivary glands and the sporozoites counting under microscope. But in area of low exposure and because few mosquitoes are infected, many mosquitoes must be caught and dissected. The salivary glands 
dissection is a tedious technique which required well trained and studious personnel. Moreover this technique cannot differentiate Plasmodium species. Another technique named CSPELISA detects the CSP (Circumsporozoite protein) parasite surface protein and is generally done on head/thorax of mosquitoes. However the CSP protein is expressed at the oocyst stage, consequently the CSP can be detected in the mosquito before the sporozoites have reached the salivary glands (until 2-3 days) [136, 137]. Therefore, this method induced a bias with an overestimation of sporozoites index [138, 139]. Other traditional epidemiological estimates mainly based on parasitological tests are very sensitive and specific allowing the determination of parasite species, but the examination of finger prick and thick blood smear is also labour intensive and time-consuming requiring well trained staff for a reliable examination [140]. To improve the measure of transmission, antibody responses against parasite proteins (CSP, AMA1, MSP1, MSP3, etc...) could be used but several studies have highlighted limits of this approach. Actually, people exposed to malaria can be seropositive during several months [141, 142], even after transmission has stopped [141] or in the context of low transmission [143]. So by using this method we are not able to distinguish old and new infection which is particularly important in the context of evaluation of the effectiveness of vector control program. Considering these limits, these serological parameters seem inappropriate to assess the malaria exposure at the individual level. Some proteomic and transcriptomic studies highlighted that the composition of Anopheles salivary glands could be modified with the presence of Plasmodium parasite [15, 144, 145]. Therefore, the development of a biomarker specific of infective bites based on the analysis of antibody response against salivary proteins should represent an alternative method to assess the parasite transmission to the human.

The principle of biomarker of infective bites is based on the use of immunogenic salivary protein like marker of transmission. The expression of some salivary proteins could be induced or regulated when the salivary glands are infected. Therefore, if one of such protein presents also immunogenic properties, we can probably use the specific immune response to this protein like a marker of transmission in human. Such a biomarker will be also particularly relevant in the context of re-emergence after malaria transmission reduction or in area of low exposure. This tool will allow focusing the intervention (vector control strategies and drugs distribution) on the most exposed and the most susceptible population.

\section{Conclusions}

In the present chapter, we have described the development of a biomarker (the An. gambiae gSG6P1 peptide) of Anopheles mosquito bites by using an original approach coupling bioinformatic tools and immuno-epidemiological assays. Then, measurements of IgG level specific to gSG6$\mathrm{P} 1$ at individual as well as population level, represent a tool/biomarker for accurately evaluate the level of human exposure to Anopheles bites and the risk of malaria in all age-classes of populations (newborns, infants, children, adults) living in various settings (very-low, low, moderate, and high malaria transmission areas) of rural, semi-urban and urban regions of Senegal, Angola and Benin. In the majority of these areas, this biomarker appeared to be promising and complementary to classical entomological methods, because it can give a reliable 
evaluation of the individual contact with anthropophilic Anopheles even if exposure to bites is low/very low (urban area). Therefore, such biomarker would be particularly relevant in places where malaria transmission is low, e.g. in foci of urban, high-altitude or seasonal malaria, and in travelersinendemic areas. This chapterhasalso shown that the availability of such abiomarker could allow the evaluation of the exposure to the main P. falciparum vectors (An. gambiae s.s., An. arabiensis, An. funestus, An. melas) in Africa where different species of malaria vector co-inhabit. One direct application of such a gSG6 peptide marker of exposure could be in the elaboration of maps representing the risk of exposure to Anopheles bites. It could represent a geographic indicator of the risks of malaria transmission and thus a useful tool for predicting malaria morbidity risk as previously described. Furthermore, it represents a powerful and reliable tool for the evaluation of the effectiveness of vector control strategies. Such an indicator could also represent an alternative to classical entomological-parasitological monitoring methods for measuring and following the effectiveness of vector control strategies used by the National Malaria Control Programmes in various settings across Africa. Finally, this biomarker approach could be similarly applied to vector-control strategies for other mosquito-borne diseases such as emergent or re-emergent arbovirus diseases and trypanosomiasis.

\section{Author details}

Papa M. Drame ${ }^{1 *}$, Anne Poinsignon ${ }^{1}$, Alexandra Marie ${ }^{1}$, Herbert Noukpo ${ }^{2}$, Souleymane Doucoure ${ }^{1}$, Sylvie Cornelie ${ }^{1,2}$ and Franck Remoue ${ }^{1,2}$

*Address all correspondence to: drpapamak@gmail.com

1 Universités Montpellier 1 et 2, Institut de Recherche pour le Développement (IRD), Montpellier, France

2 Universités Montpellier 1 et 2, Centre de Recherche Entomologique de Cotonou (CREC), Cotonou, Bénin

\section{References}

[1] Gubler DJ. Resurgent vector-borne diseases as a global health problem. Emerg Infect Dis 1998;4:442-50.

[2] WHO. 2011. In: Organization WH, ed. Geneva: World Health Organization, World Malaria Report 2011.

[3] Guerra CA, Gikandi PW, Tatem AJ, et al. The limits and intensity of Plasmodium falciparum transmission: implications for malaria control and elimination worldwide. PLoS Med 2008;5:e38. 
[4] Hay SI, Guerra CA, Gething PW, et al. A world malaria map: Plasmodium falciparum endemicity in 2007. PLoS Med 2009;6:e1000048.

[5] WHO. Global Malaria Action Plan World Health Organization. Geneva, Switzerland: World Health Organization, 2009.

[6] The MalERA Consultative group on Monitoring EaS. A research agenda for malaria eradication: monitoring, evaluation, and surveillance. PLoS Med. 2011;8:e1000400.

[7] Beier JC, Killeen GF and Githure JI. Short report: entomologic inoculation rates and Plasmodium falciparum malaria prevalence in Africa. Am J Trop Med Hyg 1999;61:109-13.

[8] Drakeley CJ, Corran PH, Coleman PG, et al. Estimating medium- and long-term trends in malaria transmission by using serological markers of malaria exposure. Proc Natl Acad Sci U S A 2005;102:5108-13.

[9] Hay SI, Rogers DJ, Toomer JF and Snow RW. Annual Plasmodium falciparum entomological inoculation rates (EIR) across Africa: literature survey, Internet access and review. Trans R Soc Trop Med Hyg 2000;94:113-27.

[10] Smith DL, Dushoff J, Snow RW and Hay SI. The entomological inoculation rate and Plasmodium falciparum infection in African children. Nature 2005;438:492-5.

[11] Clements AP, Ferry JG. Cloning, nucleotide sequence, and transcriptional analyses of the gene encoding a ferredoxin from Methanosarcina thermophila. J Bacteriol 1992;174:5244-50.

[12] Vanderberg JP, Frevert U. Intravital microscopy demonstrating antibody-mediated immobilisation of Plasmodium berghei sporozoites injected into skin by mosquitoes. Int J Parasitol 2004;34:991-6.

[13] Champagne DE. Antihemostatic strategies of blood-feeding arthropods. Curr Drug Targets Cardiovasc Haematol Disord 2004;4:375-96.

[14] Champagne DE. Antihemostatic molecules from saliva of blood-feeding arthropods. Pathophysiol Haemost Thromb 2005;34:221-7.

[15] Choumet V, Carmi-Leroy A, Laurent C, et al. The salivary glands and saliva of Anopheles gambiae as an essential step in the Plasmodium life cycle: a global proteomic study. Proteomics 2007;7:3384-94.

[16] Ribeiro JM, Francischetti IM. Role of arthropod saliva in blood feeding: sialome and post-sialome perspectives. Annu Rev Entomol 2003;48:73-88.

[17] Peng Z, Simons FE. Mosquito allergy: immune mechanisms and recombinant salivary allergens. Int Arch Allergy Immunol 2004;133:198-209. 
[18] Donovan MJ, Messmore AS, Scrafford DA, Sacks DL, Kamhawi S and McDowell MA. Uninfected mosquito bites confer protection against infection with malaria parasites. Infect Immun 2007;75:2523-30.

[19] Schneider BS, Mathieu C, Peronet R and Mecheri S. Anopheles stephensi saliva enhances progression of cerebral malaria in a murine model. Vector Borne Zoonotic Dis 2011;11:423-32

[20] Andrade BB, Rocha BC, Reis-Filho A, et al. Anti-Anopheles darlingi saliva antibodies as marker of Plasmodium vivax infection and clinical immunity in the Brazilian Amazon. Malar J 2009;8:121.

[21] Orlandi-Pradines E, Almeras L, Denis de Senneville L, et al. Antibody response against saliva antigens of Anopheles gambiae and Aedes aegypti in travellers in tropical Africa. Microbes Infect 2007;9:1454-62.

[22] Remoue F, Cisse B, Ba F, et al. Evaluation of the antibody response to Anopheles salivary antigens as a potential marker of risk of malaria. Trans R Soc Trop Med Hyg 2006;100:363-70

[23] Waitayakul A, Somsri S, Sattabongkot J, Looareesuwan S, Cui L and Udomsangpetch R. Natural human humoral response to salivary gland proteins of Anopheles mosquitoes in Thailand. Acta Trop 2006;98:66-73.

[24] Schneider BS, Higgs S. The enhancement of arbovirus transmission and disease by mosquito saliva is associated with modulation of the host immune response. Trans $\mathrm{R}$ Soc Trop Med Hyg 2008;102:400-8.

[25] Londono-Renteria BL, Eisele TP, Keating J, James MA and Wesson DM. Antibody response against Anopheles albimanus (Diptera: Culicidae) salivary protein as a measure of mosquito bite exposure in Haiti. J Med Entomol 2010;47:1156-63.

[26] Poinsignon A, Cornelie S, Mestres-Simon M, et al. Novel peptide marker corresponding to salivary protein gSG6 potentially identifies exposure to Anopheles bites. PLoS One 2008;3:e2472

[27] Drame PM, Machault V, Diallo A, et al. IgG responses to the gSG6-P1 salivary peptide for evaluating human exposure to Anopheles bites in urban areas of Dakar region, Senegal. Malar J 2012;11:72.

[28] Poinsignon A, Cornelie S, Ba F, et al. Human IgG response to a salivary peptide, gSG6-P1, as a new immuno-epidemiological tool for evaluating low-level exposure to Anopheles bites. Malar J 2009;8:198.

[29] Poinsignon A, Samb B, Doucoure S, et al. First attempt to validate the gSG6-P1 salivary peptide as an immuno-epidemiological tool for evaluating human exposure to Anopheles funestus bites. Trop Med Int Health 2010;15:1198-203. 
[30] Drame PM, Poinsignon A, Besnard P, et al. Human antibody responses to the Anopheles salivary gSG6-P1 peptide: a novel tool for evaluating the efficacy of ITNs in malaria vector control. PLoS One 2010;5:e15596.

[31] Odegaard F. How many species of arthropods? Erwin's estimate revised. Biological Journal of the Linnean Society 2000;71:583-597.

[32] Ribeiro JM. Blood-feeding arthropods: live syringes or invertebrate pharmacologists? Infect Agents Dis 1995;4:143-52.

[33] Foster WA. Mosquito sugar feeding and reproductive energetics. Annu Rev Entomol 1995;40:443-74.

[34] Ribeiro JM. Role of saliva in blood-feeding by arthropods. Annu Rev Entomol 1987;32:463-78.

[35] Francischetti IM, Sa-Nunes A, Mans BJ, Santos IM and Ribeiro JM. The role of saliva in tick feeding. Front Biosci 2009;14:2051-88.

[36] Ribeiro JM, Rossignol PA and Spielman A. Role of mosquito saliva in blood vessel location. J Exp Biol 1984;108:1-7.

[37] Champagne DE. The role of salivary vasodilators in bloodfeeding and parasite transmission. Parasitol Today 1994;10:430-3.

[38] Stark KR, James AA. Salivary gland anticoagulants in culicine and anopheline mosquitoes (Diptera:Culicidae). J Med Entomol 1996;33:645-50.

[39] Francischetti IM. Platelet aggregation inhibitors from hematophagous animals. Toxicon 2010;56:1130-44.

[40] Lombardo F, Di Cristina M, Spanos L, Louis C, Coluzzi M and Arca B. Promoter sequences of the putative Anopheles gambiae apyrase confer salivary gland expression in Drosophila melanogaster. J Biol Chem 2000;275:23861-8.

[41] Arca B, Lombardo F, Capurro M, et al. Salivary gland-specific gene expression in the malaria vector Anopheles gambiae. Parassitologia 1999;41:483-7.

[42] Arca B, Lombardo F, de Lara Capurro M, et al. Trapping cDNAs encoding secreted proteins from the salivary glands of the malaria vector Anopheles gambiae. Proc Natl Acad Sci U S A 1999;96:1516-21.

[43] Calvo E, Andersen J, Francischetti IM, et al. The transcriptome of adult female Anopheles darlingi salivary glands. Insect Mol Biol 2004;13:73-88.

[44] Calvo E, Mans BJ, Andersen JF and Ribeiro JM. Function and evolution of a mosquito salivary protein family. J Biol Chem 2006;281:1935-42.

[45] Yoshida S, Sudo T, Niimi M, et al. Inhibition of collagen-induced platelet aggregation by anopheline antiplatelet protein, a saliva protein from a malaria vector mosquito. Blood 2008;111:2007-14. 
[46] Francischetti IM, Valenzuela JG and Ribeiro JM. Anophelin: kinetics and mechanism of thrombin inhibition. Biochemistry 1999;38:16678-85.

[47] Isawa $\mathrm{H}$, Yuda M, Orito $\mathrm{Y}$ and Chinzei $\mathrm{Y}$. A mosquito salivary protein inhibits activation of the plasma contact system by binding to factor XII and high molecular weight kininogen. J Biol Chem 2002;277:27651-8.

[48] Champagne DE, Ribeiro JM. Sialokinin I and II: vasodilatory tachykinins from the yellow fever mosquito Aedes aegypti. Proc Natl Acad Sci U S A 1994;91:138-42.

[49] Ribeiro JM. Characterization of a vasodilator from the salivary glands of the yellow fever mosquito Aedes aegypti. J Exp Biol 1992;165:61-71.

[50] Ribeiro JM, Valenzuela JG. Purification and cloning of the salivary peroxidase/catechol oxidase of the mosquito Anopheles albimanus. J Exp Biol 1999;202:809-16.

[51] Fontaine A, Pascual A, Diouf I, et al. Mosquito salivary gland protein preservation in the field for immunological and biochemical analysis. Parasit Vectors 2011;4:33.

[52] Wikel SK. Immune responses to arthropods and their products. Annu Rev Entomol 1982;27:21-48.

[53] Konik P, Slavikova V, Salat J, Reznickova J, Dvoroznakova E and Kopecky J. Antitumour necrosis factor-alpha activity in Ixodes ricinus saliva. Parasite Immunol 2006;28:649-56.

[54] Titus RG, Bishop JV and Mejia JS. The immunomodulatory factors of arthropod saliva and the potential for these factors to serve as vaccine targets to prevent pathogen transmission. Parasite Immunol 2006;28:131-41.

[55] Andrade BB, Barral-Netto M. Biomarkers for susceptibility to infection and disease severity in human malaria. Mem Inst Oswaldo Cruz 2011;106 Suppl 1:70-8.

[56] Peng Z, Estelle F and Simons R. Mosquito allergy and mosquito salivary allergens. Protein Pept Lett 2007;14:975-81.

[57] Poole S, Lorenzetti BB, Cunha JM, Cunha FQ and Ferreira SH. Bradykinin B1 and B2 receptors, tumour necrosis factor alpha and inflammatory hyperalgesia. Br J Pharmacol 1999;126:649-56.

[58] Demeure CE, Brahimi K, Hacini F, et al. Anopheles mosquito bites activate cutaneous mast cells leading to a local inflammatory response and lymph node hyperplasia. J Immunol 2005;174:3932-40

[59] Billingsley PF, Baird J, Mitchell JA and Drakeley C. Immune interactions between mosquitoes and their hosts. Parasite Immunol 2006;28:143-53.

[60] Depinay N, Hacini F, Beghdadi W, Peronet R and Mecheri S. Mast cell-dependent down-regulation of antigen-specific immune responses by mosquito bites. J Immunol 2006;176:4141-6. 
[61] Gillespie RD, Mbow ML and Titus RG. The immunomodulatory factors of bloodfeeding arthropod saliva. Parasite Immunol 2000;22:319-31.

[62] Edwards JF, Higgs S and Beaty BJ. Mosquito feeding-induced enhancement of Cache Valley Virus (Bunyaviridae) infection in mice. J Med Entomol 1998;35:261-5.

[63] Schneider BS, Soong L, Girard YA, Campbell G, Mason P and Higgs S. Potentiation of West Nile encephalitis by mosquito feeding. Viral Immunol 2006;19:74-82.

[64] Schneider BS, Soong L, Zeidner NS and Higgs S. Aedes aegypti salivary gland extracts modulate anti-viral and TH1/TH2 cytokine responses to sindbis virus infection. Viral Immunol 2004;17:565-73.

[65] Esmon CT. Interactions between the innate immune and blood coagulation systems. Trends Immunol 2004;25:536-42.

[66] Bissonnette EY, Rossignol PA and Befus AD. Extracts of mosquito salivary gland inhibit tumour necrosis factor alpha release from mast cells. Parasite Immunol 1993;15:27-33.

[67] Rocha AC, Braga EM, Araujo MS, Franklin BS and Pimenta PF. Effect of the Aedes fluviatilis saliva on the development of Plasmodium gallinaceum infection in Gallus (gallus) domesticus. Mem Inst Oswaldo Cruz 2004;99:709-15.

[68] Thomson SA, Sherritt MA, Medveczky J, et al. Delivery of multiple CD8 cytotoxic T cell epitopes by DNA vaccination. J Immunol 1998;160:1717-23.

[69] Ader DB, Celluzzi C, Bisbing J, et al. Modulation of dengue virus infection of dendritic cells by Aedes aegypti saliva. Viral Immunol 2004;17:252-65.

[70] Remoue F, Alix E, Cornelie S, et al. IgE and IgG4 antibody responses to Aedes saliva in African children. Acta Trop 2007;104:108-15.

[71] Ramasamy MS, Srikrishnaraj KA, Wijekoone S, Jesuthasan LS and Ramasamy R. Host immunity to mosquitoes: effect of antimosquito antibodies on Anopheles tessellatus and Culex quinquefasciatus (Diptera: Culicidae). J Med Entomol 1992;29:934-8.

[72] Siden-Kiamos I, Louis C. Interactions between malaria parasites and their mosquito hosts in the midgut. Insect Biochem Mol Biol 2004;34:679-85.

[73] Suneja A, Gulia M and Gakhar SK. Blocking of malaria parasite development in mosquito and fecundity reduction by midgut antibodies in Anopheles stephensi (Diptera: Culicidae). Arch Insect Biochem Physiol 2003;52:63-70.

[74] Gomes R, Teixeira C, Teixeira MJ, et al. Immunity to a salivary protein of a sand fly vector protects against the fatal outcome of visceral leishmaniasis in a hamster model. Proc Natl Acad Sci U S A 2008;105:7845-50. 
[75] Brennan JD, Kent M, Dhar R, Fujioka H and Kumar N. Anopheles gambiae salivary gland proteins as putative targets for blocking transmission of malaria parasites. Proc Natl Acad Sci U S A 2000;97:13859-64.

[76] Ramamoorthi N, Narasimhan S, Pal U, et al. The Lyme disease agent exploits a tick protein to infect the mammalian host. Nature 2005;436:573-7.

[77] Dai J, Wang P, Adusumilli S, et al. Antibodies against a tick protein, Salp15, protect mice from the Lyme disease agent. Cell Host Microbe 2009;6:482-92.

[78] Peng Z, Beckett AN, Engler RJ, Hoffman DR, Ott NL and Simons FE. Immune responses to mosquito saliva in 14 individuals with acute systemic allergic reactions to mosquito bites. J Allergy Clin Immunol 2004;114:1189-94.

[79] Peng Z, Ho MK, Li C and Simons FE. Evidence for natural desensitization to mosquito salivary allergens: mosquito saliva specific IgE and IgG levels in children. Ann Allergy Asthma Immunol 2004;93:553-6.

[80] Schwartz BS, Ford DP, Childs JE, Rothman N and Thomas RJ. Anti-tick saliva antibody: a biologic marker of tick exposure that is a risk factor for Lyme disease seropositivity. Am J Epidemiol 1991;134:86-95.

[81] Schwartz BS, Ribeiro JM and Goldstein MD. Anti-tick antibodies: an epidemiologic tool in Lyme disease research. Am J Epidemiol 1990;132:58-66.

[82] Barral A, Honda E, Caldas A, et al. Human immune response to sand fly salivary gland antigens: a useful epidemiological marker? Am J Trop Med Hyg 2000;62:740-5.

[83] Nascimento RJ, Santana JM, Lozzi SP, Araujo CN and Teixeira AR. Human IgG1 and IgG4: the main antibodies against Triatoma infestans (Hemiptera: Reduviidae) salivary gland proteins. Am J Trop Med Hyg 2001;65:219-26.

[84] Drame PM, Poinsignon A, Besnard P, et al. Human antibody response to Anopheles gambiae saliva: an immuno-epidemiological biomarker to evaluate the efficacy of insecticide-treated nets in malaria vector control. Am J Trop Med Hyg 2010;83:115-21.

[85] Brosseau L, Drame PM, Besnard P, et al. Human antibody response to Anopheles saliva for comparing the efficacy of three malaria vector control methods in Balombo, Angola. PLoS One 2012;7:e44189.

[86] Ribeiro JM. A catalogue of Anopheles gambiae transcripts significantly more or less expressed following a blood meal. Insect Biochem Mol Biol 2003;33:865-82.

[87] Lombardo F, Lanfrancotti A, Mestres-Simon M, Rizzo C, Coluzzi M and Arca B. At the interface between parasite and host: the salivary glands of the African malaria vector Anopheles gambiae. Parassitologia 2006;48:573-80.

[88] Valenzuela JG. High-throughput approaches to study salivary proteins and genes from vectors of disease. Insect Biochem Mol Biol 2002;32:1199-209 
[89] Charlab R, Valenzuela JG, Rowton ED and Ribeiro JM. Toward an understanding of the biochemical and pharmacological complexity of the saliva of a hematophagous sand fly Lutzomyia longipalpis. Proc Natl Acad Sci U S A 1999;96:15155-60

[90] Holt RA, Subramanian GM, Halpern A, et al. The genome sequence of the malaria mosquito Anopheles gambiae. Science 2002;298:129-49

[91] Wang Z, Gerstein M and Snyder M. RNA-Seq: a revolutionary tool for transcriptomics. Nat Rev Genet 2009;10:57-63

[92] Jariyapan N, Roytrakul S, Paemanee A, et al. Proteomic analysis of salivary glands of female Anopheles barbirostris species A2 (Diptera: Culicidae) by two-dimensional gel electrophoresis and mass spectrometry. Parasitol Res 2012. 111(3), 1239-49.

[93] Valenzuela JG, Belkaid Y, Garfield MK, et al. Toward a defined anti-Leishmania vaccine targeting vector antigens: characterization of a protective salivary protein. J Exp Med 2001;194:331-42

[94] Arca B, Lombardo F, Valenzuela JG, et al. An updated catalogue of salivary gland transcripts in the adult female mosquito, Anopheles gambiae. J Exp Biol 2005;208:3971-86

[95] Calvo E, Dao A, Pham VM and Ribeiro JM. An insight into the sialome of Anopheles funestus reveals an emerging pattern in anopheline salivary protein families. Insect Biochem Mol Biol 2007;37:164-75

[96] Das S, Radtke A, Choi YJ, Mendes AM, Valenzuela JG and Dimopoulos G. Transcriptomic and functional analysis of the Anopheles gambiae salivary gland in relation to blood feeding. BMC Genomics 2010;11:566.

[97] Jariyapan N, Baimai V, Poovorawan Y, et al. Analysis of female salivary gland proteins of the Anopheles barbirostris complex (Diptera: Culicidae) in Thailand. Parasitol Res 2010;107:509-16.

[98] Jariyapan N, Choochote W, Jitpakdi A, et al. Salivary gland proteins of the human malaria vector, Anopheles dirus B (Diptera: Culicidae). Rev Inst Med Trop Sao Paulo 2007;49:5-10.

[99] Lombardo F, Ronca R, Rizzo C, et al. The Anopheles gambiae salivary protein gSG6: an anopheline-specific protein with a blood-feeding role. Insect Biochem Mol Biol 2009;39:457-66.

[100] Valenzuela JG, Francischetti IM, Pham VM, Garfield MK and Ribeiro JM. Exploring the salivary gland transcriptome and proteome of the Anopheles stephensi mosquito. Insect Biochem Mol Biol 2003;33:717-32.

[101] Calvo E, Pham VM, Marinotti O, Andersen JF and Ribeiro JM. The salivary gland transcriptome of the neotropical malaria vector Anopheles darlingi reveals accelerated evolution of genes relevant to hematophagy. BMC Genomics 2009;10:57. 
[102] Francischetti IM, Valenzuela JG, Pham VM, Garfield MK and Ribeiro JM. Toward a catalog for the transcripts and proteins (sialome) from the salivary gland of the malaria vector Anopheles gambiae. J Exp Biol 2002;205:2429-51.

[103] Li S, Kwon J and Aksoy S. Characterization of genes expressed in the salivary glands of the tsetse fly, Glossina morsitans morsitans. Insect Mol Biol 2001;10:69-76.

[104] Arca B, Lombardo F, Lanfrancotti A, et al. A cluster of four D7-related genes is expressed in the salivary glands of the African malaria vector Anopheles gambiae. Insect Mol Biol 2002;11:47-55.

[105] Valenzuela JG, Charlab R, Gonzalez EC, et al. The D7 family of salivary proteins in blood sucking Diptera. Insect Mol Biol 2002;11:149-55.

[106] Simons FE, Peng Z. Mosquito allergy: recombinant mosquito salivary antigens for new diagnostic tests. Int Arch Allergy Immunol 2001;124:403-5.

[107] Jariyapan N, Choochote W, Jitpakdi A, et al. A glycine- and glutamate-rich protein is female salivary gland-specific and abundant in the malaria vector Anopheles dirus B (Diptera: Culicidae). J Med Entomol 2006;43:867-74.

[108] Ribeiro JM, Arca B, Lombardo F, et al. An annotated catalogue of salivary gland transcripts in the adult female mosquito, Aedes aegypti. BMC Genomics 2007;8:6.

[109] Lanfrancotti A, Lombardo F, Santolamazza F, et al. Novel cDNAs encoding salivary proteins from the malaria vector Anopheles gambiae. FEBS Lett 2002;517:67-71.

[110] Neira Oviedo M, Ribeiro JM, Heyland A, VanEkeris L, Moroz T and Linser PJ. The salivary transcriptome of Anopheles gambiae (Diptera: Culicidae) larvae: A microarray-based analysis. Insect Biochem Mol Biol 2009;39:382-94.

[111] Okulate MA, Kalume DE, Reddy R, et al. Identification and molecular characterization of a novel protein Saglin as a target of monoclonal antibodies affecting salivary gland infectivity of Plasmodium sporozoites. Insect Mol Biol 2007;16:711-22.

[112] Shen Z, Jacobs-Lorena M. A type I peritrophic matrix protein from the malaria vector Anopheles gambiae binds to chitin. Cloning, expression, and characterization. J Biol Chem 1998;273:17665-70.

[113] Calvo E, Pham VM, Lombardo F, Arca B and Ribeiro JM. The sialotranscriptome of adult male Anopheles gambiae mosquitoes. Insect Biochem Mol Biol 2006;36:570-5.

[114] Isawa H, Orito $Y$, Iwanaga S, et al. Identification and characterization of a new kallikrein-kinin system inhibitor from the salivary glands of the malaria vector mosquito Anopheles stephensi. Insect Biochem Mol Biol 2007;37:466-77.

[115] Arca B, Lombardo F, Francischetti IM, et al. An insight into the sialome of the adult female mosquito Aedes albopictus. Insect Biochem Mol Biol 2007;37:107-27. 
[116] Ribeiro JM, Charlab R, Pham VM, Garfield M and Valenzuela JG. An insight into the salivary transcriptome and proteome of the adult female mosquito Culex pipiens quinquefasciatus. Insect Biochem Mol Biol 2004;34:543-63.

[117] Cornelie S, Remoue F, Doucoure S, et al. An insight into immunogenic salivary proteins of Anopheles gambiae in African children. Malar J 2007;6:75.

[118] Rizzo C, Ronca R, Fiorentino G, et al. Humoral response to the Anopheles gambiae salivary protein gSG6: a serological indicator of exposure to Afrotropical malaria vectors. PLoS One 2011;6:e17980.

[119] Rizzo C, Ronca R, Fiorentino G, et al. Wide cross-reactivity between Anopheles gambiae and Anopheles funestus SG6 salivary proteins supports exploitation of gSG6 as a marker of human exposure to major malaria vectors in tropical Africa. Malar J 2011;10:206.

[120] Larru B, Molyneux E, Ter Kuile FO, Taylor T, Molyneux M and Terlouw DJ. Malaria in infants below six months of age: retrospective surveillance of hospital admission records in Blantyre, Malawi. Malar J 2009;8:310.

[121] Rogier C. [Childhood malaria in endemic areas: epidemiology, acquired immunity and control strategies]. Med Trop (Mars) 2003;63:449-64.

[122] Carnevale P, Frezil JL, Bosseno MF, Le Pont F and Lancien J. [The aggressiveness of Anopheles gambiae A in relation to the age and sex of the human subjects]. Bull World Health Organ 1978;56:147-54.

[123] Geissbuhler Y, Chaki P, Emidi B, et al. Interdependence of domestic malaria prevention measures and mosquito-human interactions in urban Dar es Salaam, Tanzania. Malar J 2007;6:126.

[124] Drame PM, Diallo A, Poinsignon A, et al. Evaluation of the effectiveness of malaria vector control measures in urban settings of Dakar by a specific Anopheles salivary biomarker. PLoS One (2013). In press.

[125] Palmeira P, Quinello C, Silveira-Lessa AL, Zago CA and Carneiro-Sampaio M. IgG placental transfer in healthy and pathological pregnancies. Clin Dev Immunol 2012;2012:985646.

[126] Kinyanjui SM, Bull P, Newbold CI and Marsh K. Kinetics of antibody responses to Plasmodium falciparum-infected erythrocyte variant surface antigens. J Infect Dis 2003;187:667-74.

[127] Gadiaga L, Machault V, Pages F, et al. Conditions of malaria transmission in Dakar from 2007 to 2010. Malar J 2011;10:312.

[128] Machault V, Gadiaga L, Vignolles C, et al. Highly focused anopheline breeding sites and malaria transmission in Dakar. Malar J 2009;8:138. 
[129] Kalluri S, Gilruth P, Rogers D and Szczur M. Surveillance of arthropod vector-borne infectious diseases using remote sensing techniques: a review. PLoS Pathog 2007;3:1361-71.

[130] Smith T, Killeen G, Lengeler C and Tanner M. Relationships between the outcome of Plasmodium falciparum infection and the intensity of transmission in Africa. Am J Trop Med Hyg 2004;71:80-6.

[131] Corran P, Coleman P, Riley E and Drakeley C. Serology: a robust indicator of malaria transmission intensity? Trends Parasitol 2007;23:575-82

[132] Doannio JM, Doudou DT, Konan LY, et al. [Influence of social perceptions and practices on the use of bednets in the malaria control programme in Ivory Coast (West Africa)]. Med Trop (Mars) 2006;66:45-52.

[133] Pages F, Orlandi-Pradines E and Corbel V. [Vectors of malaria: biology, diversity, prevention, and individual protection]. Med Mal Infect 2007;37:153-61.

[134] Corbel V, Akogbeto M, Damien GB, et al. Combination of malaria vector control interventions in pyrethroid resistance area in Benin: a cluster randomised controlled trial. Lancet Infect Dis 2012;12:617-26.

[135] Okumu FO, Moore SJ. Combining indoor residual spraying and insecticide-treated nets for malaria control in Africa: a review of possible outcomes and an outline of suggestions for the future. Malar J 2011;10:208.

[136] Beier JC. Malaria parasite development in mosquitoes. Annu Rev Entomol 1998;43:519-43.

[137] Lombardi S, Esposito F, Zavala F, et al. Detection and anatomical localization of Plasmodium falciparum circumsporozoite protein and sporozoites in the Afrotropical malaria vector Anopheles gambiae s.l. Am J Trop Med Hyg 1987;37:491-4.

[138] Burkot TR, Williams JL and Schneider I. Identification of Plasmodium falciparum-infected mosquitoes by a double antibody enzyme-linked immunosorbent assay. Am J Trop Med Hyg 1984;33:783-8.

[139] Fontenille D, Meunier JY, Nkondjio CA and Tchuinkam T. Use of circumsporozoite protein enzyme-linked immunosorbent assay compared with microscopic examination of salivary glands for calculation of malaria infectivity rates in mosquitoes (Diptera: Culicidae) from Cameroon. J Med Entomol 2001;38:451-4.

[140] Siala E, Ben Abdallah R, Bouratbine A, Aoun K. Current biological diagnosis of malaria. Revue Tunisienne d'Infectiologie 2010;4:5-9.

[141] Druilhe P, Pradier O, Marc JP, Miltgen F, Mazier D and Parent G. Levels of antibodies to Plasmodium falciparum sporozoite surface antigens reflect malaria transmission rates and are persistent in the absence of reinfection. Infect Immun 1986;53:393-7. 
[142] Greenhouse B, Ho B, Hubbard A, et al. Antibodies to Plasmodium falciparum antigens predict a higher risk of malaria but protection from symptoms once parasitemic. J Infect Dis 2011;204:19-26.

[143] Clark EH, Silva CJ, Weiss GE, et al. Plasmodium falciparum malaria in the Peruvian Amazon, a region of low transmission, is associated with immunologic memory. Infect Immun 2012;80:1583-92.

[144] Dixit R, Sharma A, Mourya DT, Kamaraju R, Patole MS and Shouche YS. Salivary gland transcriptome analysis during Plasmodium infection in malaria vector Anopheles stephensi. Int J Infect Dis 2009;13:636-46.

[145] Rosinski-Chupin I, Briolay J, Brouilly P, et al. SAGE analysis of mosquito salivary gland transcriptomes during Plasmodium invasion. Cell Microbiol 2007;9:708-24. 
